\title{
THE INFLUENCE OF ATTENTIVE FIXATION UPON THE EXCITABILITY OF THE LIGHT-SENSITIVE NEURONS OF THE POSTERIOR PARIETAL CORTEX ${ }^{1}$
}

\author{
VERNON B. MOUNTCASTLE, ${ }^{2}$ RICHARD A. ANDERSEN, AND BRAD C. MOTTER \\ Department of Physiology, The Johns Hopkins University School of Medicine, Baltimore, Maryland 21205
}

\begin{abstract}
We describe the effect of behavioral state upon the excitability of light-sensitive (LS) neurons of the inferior parietal lobule, area 7a, studied in waking monkeys. The responses of parietal LS neurons to visual stimuli are facilitated during the state of attentive fixation of a target light as compared to their responses to physically and retinotopically identical test stimuli delivered during the eye pauses of alert wakefulness. Seventy percent of the neurons tested $(n=55)$ showed significant increments in responses in the state of attentive fixation; the median value of the increments was 3.5 times. Only 4 of the 55 cells examined completely showed the reverse relation. Three sets of control experiments were done. The facilitation occurred when the responses evoked during the trials of a reaction task with attentive fixation of a target were compared with those evoked by identical stimuli delivered to the same retinotopic locations at the end of each intertrial interval: the facilitation of attentive fixation is not due to a shift in the general level of arousal. The facilitation occurred when the animal maintained attentive fixation of a spot on the tangent screen without a target light or when an additional light mimicking the target light was presented along with testing stimuli in the state of alert wakefulness without attentive fixation: the facilitation is not produced by a sensory-sensory interaction between target and testing lights. Finally, the facilitation was observed whether or not the test stimuli were behaviorally relevant.

We conclude that the act of attentive fixation exerts a specific and powerful effect upon the excitability of the neural systems linking the retinae and the inferior parietal lobule and that the facilitation plays an important role in visually guided behavior.
\end{abstract}

The functional properties of the neurons of the inferior parietal lobule of the cerebral cortex of the waking monkey have been surveyed in a number of studies. There is agreement that one large class of parietal neurons is sensitive to visual stimuli. We recently studied the functional properties of those light-sensitive cells in a behavioral state that we define as interested or attentive fixation (Motter and Mountcastle, 1981). A monkey working in this state fixates a small target whose dimming he must detect for liquid reward. Test visual stimuli delivered during these periods of fixation are passive probes, for they do not control behavior. We discovered that the excitability of parietal light-sensitive (LS) neurons is greatly increased during periods of target fixation in this detection task, as compared to that in periods of eye pauses without attentive fixation, as the monkey sat quietly. These observations are described in the present paper. Our conclusion is that the behavioral state of

'This work was supported by Grant 5 RO 1 EY03517 from the United States Public Health Service which we gratefully acknowledge.

${ }^{2}$ To whom correspondence and reprint requests should be addressed. attentive fixation is associated with an increase in excitability in the neural systems linking the retinae with the parietal cortex. Moreover, we found that the increased responsiveness of the system during attentive fixation, like the functional properties of LS neurons, is unchanged when attentive fixation is maintained in the absence of a visual target.

The excitability of parietal LS neurons also is influenced by the angle of gaze. Evidence concerning that phenomenon will be presented in a future paper (R. A. Andersen and V. B. Mountcastle, manuscript in preparation) together with descriptions of the interactions between these two control states.

Two short notes have appeared previously (Motter and Mountcastle, 1980; Andersen and Mountcastle, 1980).

\section{Materials and Methods}

\section{General description}

The data analyzed were drawn from an experimental series of 10 monkeys described in a recent paper (Motter 
and Mountcastle, 1981) with the addition of one animal trained for the special control experiment described below. The behavioral and electrophysiological methods that we used are described in previous publications (Motter and Mountcastle, 1981; Mountcastle et al., 1975; Lynch et al., 1977). Briefly, macaque monkeys sat in a light-tight enclosure viewing binocularly a $127^{\circ} \times 150^{\circ}$ tangent screen. Trained animals achieved and maintained fixations for periods of 0.5 to $5.0 \mathrm{sec}$ of a $0.3^{\circ}$ target light generated by a laser and positioned and moved on the screen by mirror galvanometers. The monkeys held the eye position even though other visual stimuli were delivered during the fixation periods and gained liquid reward for detecting dimming of the target light, which followed the stimulus presentation. Luminous test stimuli were back-projected onto the tangent screen; the projected images could be varied in size and shape (Fig. 1, task mode A). They were usually 0.2 to 0.6 $\log$ unit in intensity above a background of 1 to $2 \mathrm{~cd} / \mathrm{m}^{2}$, but intensities up to $2 \log$ units were available and occasionally used. Stimuli could be positioned anywhere on the screen, turned on and off, and moved in any direction at velocities up to $600^{\circ} / \mathrm{sec}$ under program control.

Alternatively, a $25 \times 27$ matrix of light-emitting diodes (LEDs) on 2-cm centers could be inserted 34 to $50 \mathrm{~cm}$ in front of the animal. Individual LEDs could be turned on or off in any sequence, under program control, except that only two LEDs could be on simultaneously. Runs in which saccadic movements were induced by shifts in position of the target light were used also. Monkey and tangent screen were observed via normal and infrared video monitoring. The sequence of stimulus and behavioral events, the collection and storage of signals of neural activity, and the electro-oculographic records of eye position were controlled by a PDP 11-20 computer.

In addition to the regular behavioral task outlined above, monkey 88 was trained to perform a second task which required him to maintain fixation upon the locus of the fixation target during a 2- to 3-sec "off" period in which the light was extinguished (Fig. 1, task mode B). Trials were terminated if the eyes moved during that period.

The electrical signs of the impulse discharge of single cortical neurons were recorded from extracellular positions with Pt-Ir, glass-coated electrodes passed via hydraulically closed cranial chambers through the intact dura into the parietal cortex. Usually one microelectrode penetration was made during each day's recording session of 6 to $7 \mathrm{hr}$. The cranial chamber then was closed and the animal was returned to its living cage overnight. Recording continued for 2 to 4 weeks in each hemisphere. All neurons considered here were identified positively as light sensitive in computer-controlled runs with projected test stimuli. The locations of some microelectrode penetrations were determined in the manner described earlier (Motter and Mountcastle, 1981); all were shown to have passed into area 7 of the inferior parietal lobule. Conventional spike train analyses were used off-line for studies of the time relations between neuronal discharges, behavioral events, and eye position and movement. Special analyses are described below.

\section{Definitions of behavioral states}

We describe below changes in the excitability of parietal LS neurons and correlate them with the behavioral conditions occurring at the time of stimulus delivery. Differences between the sets of behavioral conditions are not easily described with respect to a common, behaviorally measurable continuum. For clarity, we elect to define in operational terms a behavioral state or "mode" for each of the four sets of behavioral conditions that we have studied.

Task A state: A state of interested or attentive fixation of a visible target light. A monkey working in this state captured visually a small $\left(0.2^{\circ}\right.$ to $\left.0.3^{\circ}\right)$ target light when it appeared on a tangent screen, maintained fixation for foreperiods of 0.5 to $5 \mathrm{sec}$ duration, and detected the dimming of the target light to earn liquid reward. The times of dimming could not be anticipated, for both the intertrial intervals and foreperiods of different lengths occurred in pseudorandom order. Electro-oculographic recording and programmatic control provided trial interruption and an error signal (modulated sound) if the eye moved more than a selected distance from the target during the foreperiod. We define this state of maintained fixation coupled with a high probability of correct detection as one of interested fixation or directed visual attention. Testing visual stimuli were delivered during the foreperiods (see Fig. 1).

Task $B$ state: $A$ state of interested fixation in the absence of a visible target light. One monkey was trained to maintain fixation of the locus of the target light, even when it was turned off and then on again during the foreperiod, and to detect its dimming at the end of the foreperiod. Testing visual stimuli were delivered during the period of steady fixation of target locus without target light. We define this as a state of directed visual attention like that above.

No task state: An alert but quiescent state with no involvement (participation) in any behavioral task. The animal in this state sat facing the tangent screen; no manipulanda were available. His alertness was suggested by electro-oculographic signs of successive eye pauses and brisk saccadic movements. Test visual stimuli were delivered to different loci on the tangent screen in pseudorandom order, and the responses to those delivered during eye pauses were compared with the responses evoked by physically and retinotopically identical stimuli delivered in task states $\mathrm{A}$ and $\mathrm{B}$.

Intertrial state: An alert, perhaps anticipatory state, occurring between the trials of task $A$. The onset of the target light at the end of each intertrial interval provided a test of the excitability of LS neurons in another behavioral state. Intertrial intervals varied randomly in duration, as did the onset positions of the target light, so that neither the time nor the location of the target light at the beginning of a trial could be anticipated. The target light at the onset was thus a visual test stimulus. This permitted correlations between stimulus and eye positions and neuronal responses. We term this the intertrial state and infer that it resembles the level of general vigilance and arousal characteristic of the task states, for in it, the animal reacted to the target onset with brisk 
TASK MODE -A

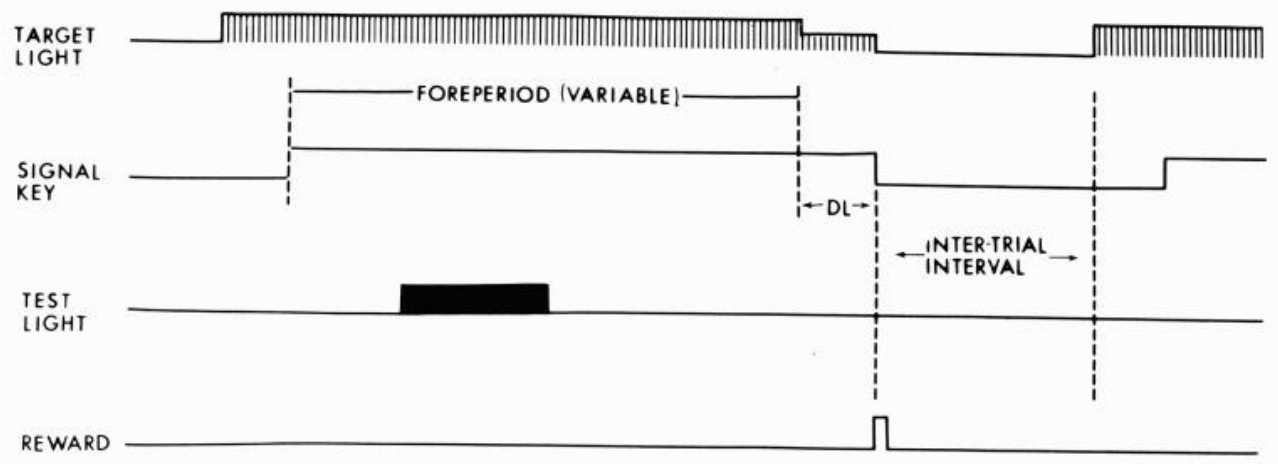

TASK MODE - B

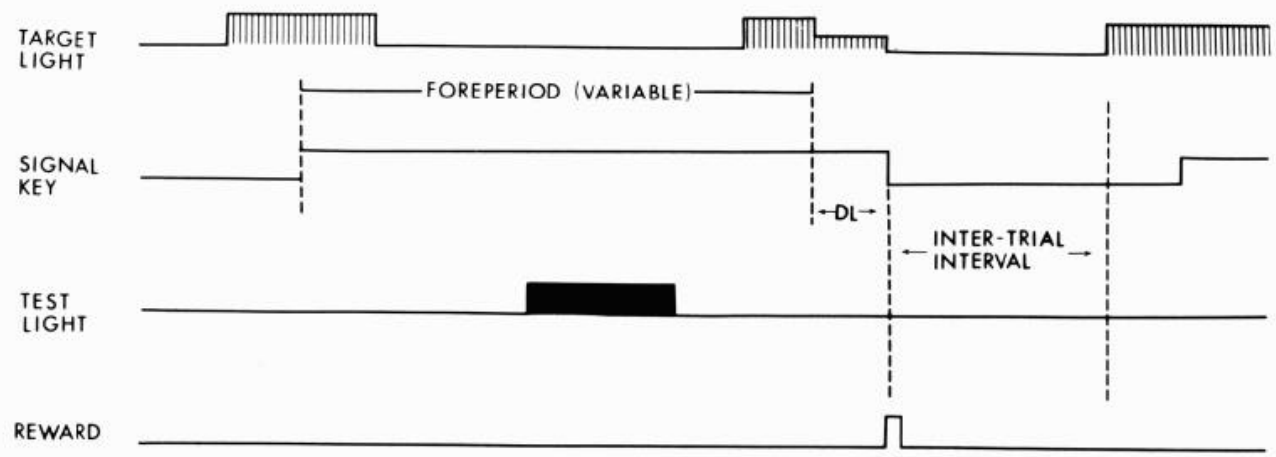

SEC

Figure 1. Course of events in task modes $A$ and $B$. A trial in task mode $A$ began with the fixation target light onset and progressed if the subject closed the signal key within a pre-set time period. A variable foreperiod followed during which testing light stimuli were delivered back-projected anywhere upon the central $100^{\circ} \times 100^{\circ}$ area of a $127^{\circ} \times 150^{\circ}$ tangent screen and centered on the fixation point. At the end of the foreperiod, the fixation stimulus dimmed slightly; if the subject detected that dimming and released the signal key within a pre-set time, he received a drop of liquid reward. Visual stimuli with different parameters were delivered in a random order from trial to trial, up to 10 classes per run. Break of fixation or premature key release during a foreperiod caused termination of that trial, delivery of a 500 -msec burst of noise, and a punishment delay; the program then entered the intertrial interval leading to the next trial. Task mode $B$ was identical except that the fixation light was extinguished before, during, and immediately after delivery of the test visual stimulus during the foreperiod and reappeared later before dimming, etc. The animal trained in this task maintained fixation of the location at which the fixation target would reappear during its blackout. A break in fixation led to the error signal and termination of the trial as above. $D L$, dimming of the target light.

target-capturing saccades and a motor response to close a signal key. The intertrial period differs from the task states, for in it, the target light at the onset appears as a testing visual stimulus at a time of eye pause without attentive fixation and when no other targets or test lights are present in the field (see Fig. 8). The intertrial state differs also from the no task state, for in the former but not the latter, the animal alertly awaits and reacts to a trial onset.

\section{Special methods of analysis}

Comparison of responses in the task and no task states. A comparison was made between the task and no task conditions by stimulating the same retinotopic locus with identical stimuli in the two states. The retinal positions of the stimuli in the no task state were calculated from eye position and stimulus location information collected at the time of stimulation, and those in the task mode were determined by the fixation positions dictated by the task itself. The visual stimuli delivered in the no task state appeared at positions on the screen which changed in a pseudorandom order from trial to trial. The neural responses to these stimuli were collected under computer control. A calibration of the electro-oculograms (EOGs) was performed just before each no task run (Fig. 2). Horizontal or vertical signals sometimes appeared in 
the inappropriate channel; they were removed by a hardware subtraction. The coefficients of variation of final eye positions after $20^{\circ}$ deviations ranged from 2 to $5 \%$. Voltage values measured in the calibration runs were used to calibrate the measurements of the eye positions in the no task state. Each quadrant was treated separately, using the appropriate values from the calibration run.

The records chosen for analysis in the no task condition met certain criteria for steady eye pauses at and around the time of stimulation. For all of the analyses presented here, trials were rejected if the animal made a single saccade of $2^{\circ}$ or a series of small repeated saccades that summed to $5^{\circ}$ during a 500 - to 1100 -msec period centered on the 100 - to 200 -msec stimulus. Figure 3 shows on the left examples of the horizontal EOG traces of trials selected for further analysis and, on the right, those rejected-all from a single run in the no task condition. However, we found no significant differences in the results for the different eye pause periods analyzed.

The $x-y$ plot at the upper left of Figure 4 shows the fixation positions on the tangent screen in those trials selected for analysis in a particular run. The origin of the axes is the point on the screen corresponding to straight ahead fixation. This animal tended to look upward in the no task condition. In this run, 80 records of 252 trials collected passed our criteria for stability of eye position at the time of stimulus presentation. These data are transformed to construct the plot to the lower left of Figure 4 (by subtracting eye position from stimulus position), where each cross represents the retinotopic position of the stimulus with reference to the fixation point. The dashed line box in this plot encloses those positions at which stimuli evoked the responses summed in the histogram to the left in Figure 4 in the no task mode. The histogram to the right sums the responses to stimuli delivered at the same retinotopic position but now, as the animal attentively fixated a target light, working in task mode A.

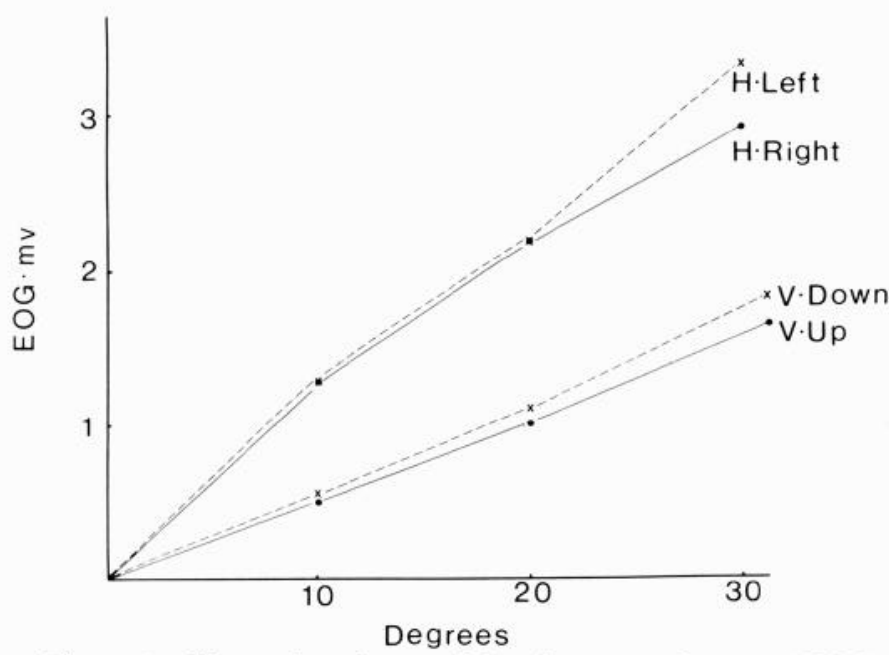

Figure 2. Change in voltage of the electro-oculogram (EOG) as a function of eye position in a monkey subject. The EOG was recorded via $\mathrm{Ag}-\mathrm{AgCl}$ electrodes implanted in the orbital rims. The function was assumed to be linear for analyses. The abbreviations used in this and the following figures are: $\mathrm{mv}$, millivolt; $\mathrm{H}$, horizontal EOG; V, vertical EOG.

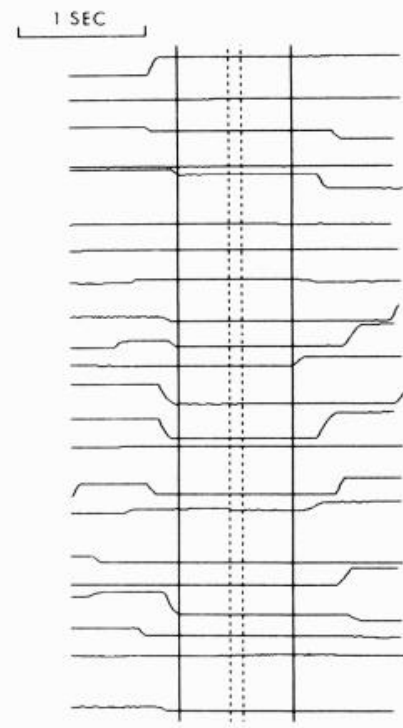

SELECTED TRIALS

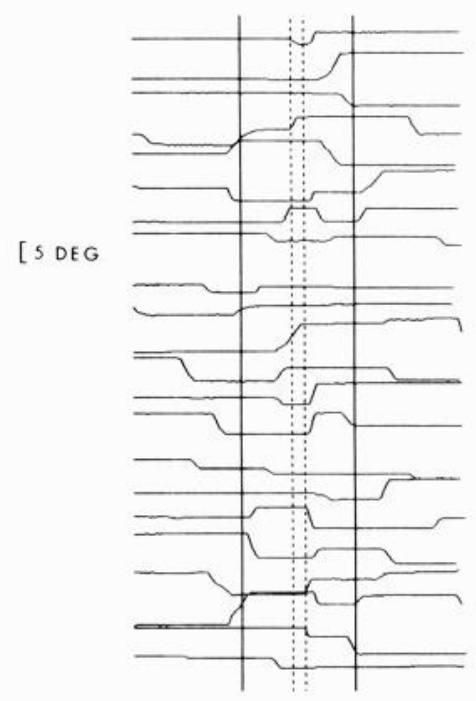

REJECTED TRIALS
Figure 3. Examples of horizontal electro-oculograms selected and those rejected for the analyses of the responses evoked by visual stimuli delivered in the no task mode (see Fig. 4). Vertical dashed lines indicate 100-msec periods of stimulation. Vertical solid lines indicate criterion limits for eye pause, here of $900 \mathrm{msec}$ duration. Further analyses showed that the response differentials were similar when the pre- and poststimulus eye pause requirements were reduced to $200 \mathrm{msec}$ each.

Standard statistical methods were used to test the significance of putative responses, as compared to the spontaneous activity immediately preceding the stimuli, and the differences between the histograms of responses in different behavioral states. The methods used are indicated in the figure legends.

Comparison of the responses evoked in the intertrial period with those during the trial periods. The responses of neurons to the onset of the fixation target at the end of the intertrial interval were studied for two purposes. Firstly, it was reasoned that this response occurred at a general level of arousal/vigilance which resembled that of the task state more closely than did that of the no task state. Secondly, it allowed a comparison between the responses to behaviorally relevant stimuli when matched with the responses evoked by saccade targets during the trial period; each of the stimuli compared evoked an eye movement to capture and fixate identical targets. All stimuli and targets were physically identical for any one comparison, either LEDs or laser spots. The retinotopic locations of stimuli delivered during trial periods were apparent because the direction of gaze was dictated by the fixation point. The retinotopic location of this fixation target, when serving as a test stimulus at the trial onset, was calculated from the $x-y$ vector difference between the eye position at the time of target onset and the eye position later during target fixation. The problem of EOG drift was eliminated because the retinotopic positions were determined for each trial by the change in eye position required to fixate the test stimuli. Comparisons then could be made between the responses to physically identical stimuli appearing in similar retinotopic positions under two different behavioral conditions. 
The monkeys were trained to orient quickly to the fixation target; trials were rejected from our comparison sets if the eyes moved within $100 \mathrm{msec}$ of target onset. Figures 8,9 , and 10 show acceptable trials ordered according to the latencies from the target onsets to the first eye movements which occurred thereafter. Responses are plotted in a raster format with a summarizing histogram beneath each raster. Statistical comparisons were not made because of the heterogeneity of the trials caused by the different latencies of the eye movements.

\section{Results}

The data base. The data analyzed in different behavioral states were obtained in a previous study of parietal light-sensitive neurons (see Tables I and II of Motter and Mountcastle, 1981) plus the results obtained in one additional monkey, BM 88. A total of 564 LS neurons were identified in these experiments, and 418 of these were studied in task state A. One hundred seventy-five of the latter were studied also in one or more of the three other behavioral states defined above. The selection of neurons for analysis was determined only by the extent of the study in each case, particularly the adequacy of coverage of the relevant area of the retina.

Response properties of parietal light-sensitive neurons. There are three distinctive properties of parietal light-sensitive (LS) neurons when assessed during the performance of the dimming detection task, i.e., in task mode A (Motter and Mountcastle, 1981). Firstly, the majority are related to large response areas frequently distributed in both halves of the visual fields but sparing the central zone of vision (foveal sparing). The response areas of the minority do include the foveal and the perifoveal regions, when tested with relatively large stimuli $\left(6^{\circ} \times 6^{\circ}\right)$, but only 8 of 261 cells studied in detail by Motter and Mountcastle (1981) responded to the small fixation light. Secondly, parietal LS neurons are sensitive to stimulus movement over a range from 3 to $600^{\circ} / \mathrm{sec}$, usually with flat velocity-response functions. This movement sensitivity is strongly directional. Thirdly, the movement vectors of LS neurons with bilateral response areas point in opposite directions in the two halves of the visual fields, either inward loward the central line of gaze (75\%) or outward toward the rims of the visual fields. We term this opponent vector organization. These large bilateral response areas commonly include the monocular crescents, and a few are confined to those far lateral regions of the visual fields.

The facilitation of the response of parietal light-sensitive neurons to visual stimuli during attentive fixation. The facilitation of the responses of an LS neuron to moving visual stimuli delivered during periods of attentive fixation is illustrated in Figure 4 for comparison with those evoked in a state of quiet alertness without attentive fixation. The $x-y$ plot at the upper left shows eye positions at the time of the stimulus onset for trials selected for the stability of eye position before, during, and after stimulus delivery as illustrated in Figure 3 and described under "Materials and Methods." The animal sat quietly in the no task behavioral state. His alertness was monitored by video inspection and was evidenced in the electro-oculographic record by brisk saccadic eye movements between eye pauses. The $x-y$ plot at the lower left was constructed by subtracting the coordinates of each eye pause from those of the start position of each accompanying stimulus. The eye pause positions thus are converted to the origin of the graph in Figure 4, lower left, where crosses mark the start positions of stimuli in relation to those eye positions. The records shown in the column headed no task mode collected under these conditions were those evoked by stimuli whose start positions are marked by the crosses within the dashed line rectangle, Figure 4 , lower left. The impulse density histogram shows that no significant neural response was evoked by these stimuli in the no task mode. Replicas of the responses evoked by physically and retinotopically identical stimuli delivered during the periods of fixation of trials as the monkey worked in the detection task are shown in the column headed task mode $A$ of Figure 4 and are summed by the impulse density histogram below. The contrast between the two sets of responses is absolute: the intense responses evoked in task A do not occur in the no task condition, even though physically identical stimuli were delivered at the same retinotopic locations in the two cases.

Some uncertainty remains concerning the spatial identity of the sets of stimuli evoking the two sets of responses compared in Figure 4. All trials selected in the task state began at the same spot and traversed the same paths, with only instrumental error of stimulus positioning to produce any scatter; that error is small. The trials selected for the no task population all began within the trajectory space of the task state stimuli, but some of their own trajectories extended outside of that pathway. If the neuron under study subtended a small response area, and particularly if that area were flanked by inhibitory side bands, the results of Figure 4 might be explained on grounds other than the differences in behavioral state. We regard this as highly unlikely, for this neuron was related to a large response area that included the region of the fovea and extended along the horizontal and vertical meridians for more than $20^{\circ}$ from the fixation point. No stimuli at any location within this large area or outside of it ever evoked inhibition of the activity of this neuron. Many trials were collected in the no task state so that comparisons could be made between the two states for stimuli delivered at a number of positions within the response area. Figure 5 provides comparisons of the summing histograms of the responses evoked at a series of retinotopically identical zones in the two states. The result shows that the zone of the facilitatory effect of attentive fixation includes a large part of the response area of the neuron; its size indicates that the differences observed cannot be explained by spatial jitter between the two sets of stimuli evoking the two sets of responses compared in the two behavioral states.

We have completed analyses like those of Figure 4 for 55 parietal LS neurons. Fifty of these cells showed incremented responses evoked by light in the task state as compared to those of the no task state, and for 38 of these, the differences were significant at the $5 \%$ level of significance ( $t$ test). For 4 LS neurons, the reverse relation held; the response in the no task mode was greater than in the task mode, and for 1 cell, there was no 

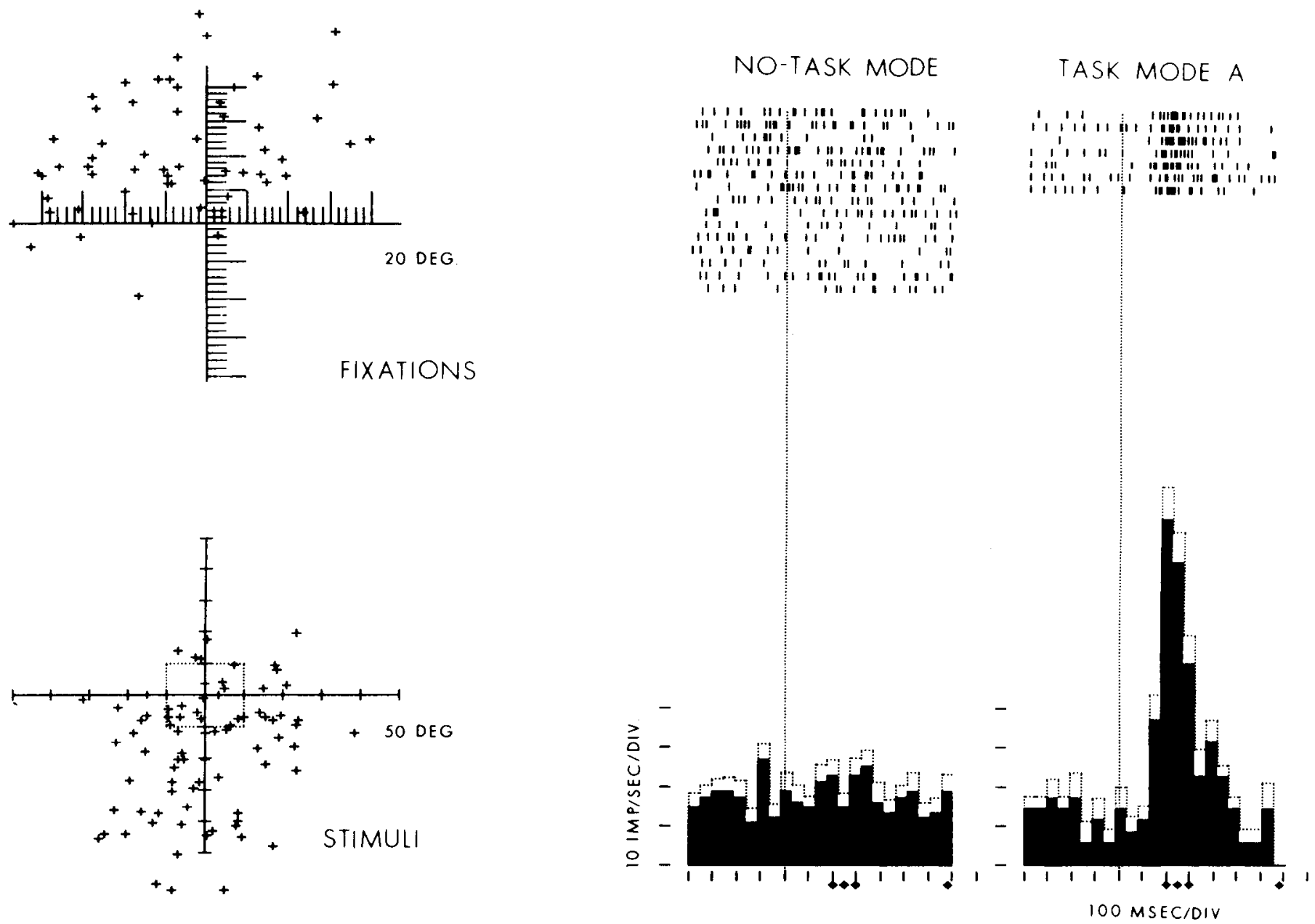

Figure 4. A comparison of the responses of parietal light-sensitive neurons to visual stimuli in the task and no task modes (states). The upper left axes show directions of gaze during eye pauses in the no task state. The center of the axes corresponds to dead-ahead fixation. Eye positions during pauses in 80 of the 252 trials of this run are plotted, for they met the requirement that each persist for $1100 \mathrm{msec}$ (see Fig. 3). Each cross on the lower left axes shows the stimulus position relative to the line of gaze during the eye pause of each trial, obtained by subtracting the eye position from that of the stimulus for each trial. Each cross marks the starting position of an $18^{\circ}$ movement of the stimulus from left to right, at $60^{\circ} / \mathrm{sec}$. The box outlined by dashes encloses the start positions of those stimuli evoking the responses in the no task mode which are shown in impulse replicas in the middle panel and summed in the histogram below. Stimuli delivered in the task mode, during steady fixation of the central point of the axes, started at the fixation point in the center of the dashed box and moved from left to right for $18^{\circ}$ at $60^{\circ} /$ sec. The responses that they evoked are shown as replicas in the panel to the right with a summing histogram below. All stimuli were $6^{\circ}$ square white lights back-projected upon a tangent screen placed $34 \mathrm{~cm}$ from the animal's eyes. The absence of responses during the task mode as compared to the strong responses in the no task mode is obvious. The standard error of the mean was calculated for each bin of the histograms; its value is shown by the dotted line above each cell of the histograms. Corresponding bin pairs within the two histograms were tested for significant differences ( $t$ test); bin pairs marked with diamonds differed at the $5 \%$ level of significance. The overall response in the two states was compared in the following way. The rate of impulse discharge in the prestimulus period was subtracted from that in the post-stimulus period for each trial, and the populations of remainders were tested for significant differences ( $p<0.05$ required) and used to form a facilitation ratio for each neuron. The ratios for neurons with significant differences are plotted in Figure 7. The units of measure used on these and the following histograms are: IMP/SEC/ DIV, impulses/sec/division; MSEC/DIV, msec/division.

difference in the two sets of responses. The results obtained for a number of cells are given in Figure 6. The differences can be expressed as a ratio of the net increment in the number of impulses in the task state compared to that in the no task state. The histogram of Figure 7 shows the distribution of this facilitation index for parietal LS neurons whose responses differed significantly.

We found no correlation between the presence or the degree of facilitation in the task mode and any other functional property of parietal LS neurons. The population of cells whose responses were facilitated during interested attention included cells with foveal and others with non-foveal response areas, those sensitive to both standing contrasts and moving stimuli, and those sensitive to movement only. The population contained cells located in each of the cellular layers of the cerebral cortex.

The field study of Figure 5 is that of an example in which stimuli delivered anywhere in the visual field were 


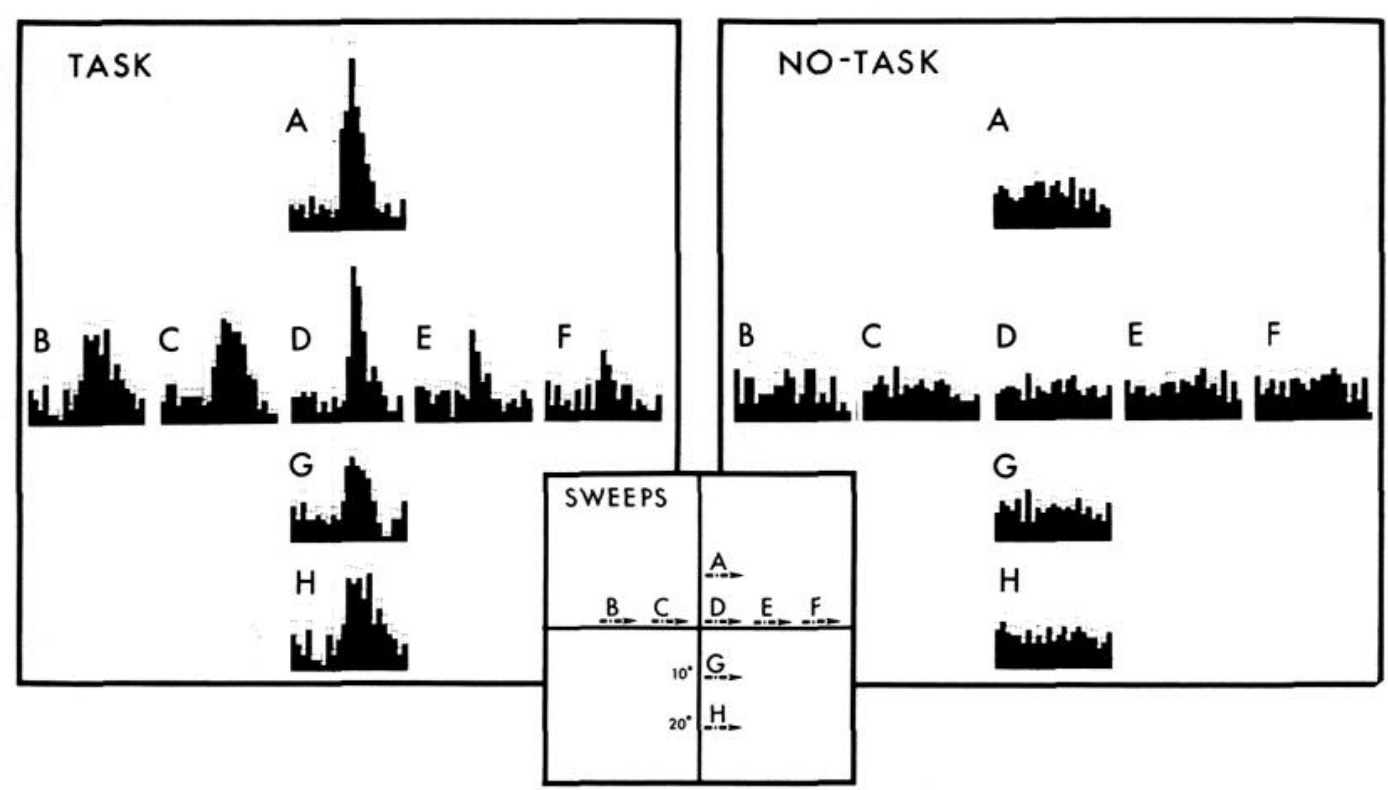

Figure 5. The facilitation of attentive fixation extends over the receptive field of parietal lightsensitive neurons. The results of further study of the neuron of Figure 4 are given; here, comparisons are shown for the responses to stimuli positioned at eight locations along the horizontal and vertical meridians (see inset). Stimulus characteristics, data collection and selection, and methods of analysis are described in the legend to Figure 4. This neuron could be activated in the task state from a large, bilateral continuous receptive field that included the region of the fixation point as tested with $6^{\circ} \times 6^{\circ}$ stimuli. It did not respond at all to physically identical stimuli delivered in those same retinotopic locations in the no task state. The histograms for point $D$ are repeated from Figure 4 .

ineffectual in the no task mode. More commonly, the difference between the two cases was relative as shown by some of the histograms of Figure 6 . We observed in these cases that the response areas determined in the no task mode usually shrank in a symmetric way as the magnitude of the response fell. However, for some other cells, nonlinear changes in field distribution were observed, but we have not yet studied these in any detail.

We observed for many neurons that the rate of spontaneous activity in the periods immediately preceding stimuli differed in the behavioral states studied, but we observed no regularity in this difference and no dependent relation between changes in spontaneous activity and differences in the responses to visual stimuli in different states.

Comparison of the excitability of parietal visual neurons in the trial and intertrial periods of the task state. We conclude from the observations just described that the increase in the excitability of parietal light-sensitive neurons in the task state as compared with the no task state accompanies the state of interested fixation, which we infer to be accompanied by directed attention. It might be supposed, on the contrary, that this difference reflects a more general change in the level of arousal, of which directed attention and the change in excitability of parietal visual neurons are concomitants without causal relation. We tested these alternatives by comparing the excitability of LS neurons in the trial periods of the task state with that occurring between trials in that same state as the monkey awaited the appearance of the fixation target. Our monkey subjects made saccadic eye movements to this light at the trial onset at latencies and with accuracies comparable to those of saccadic movements from one target to another during the trial period. We infer from this that the general level of arousal or vigilance was more or less the same throughout the working runs of the task state that include rapidly alternating states of attentive fixation between trial and intertrial periods. These latter are of comparable or shorter durations than the trial periods, are filled with eye pauses and spontaneous saccades, and are terminated at the trial onset by saccadic capture of the target light and a reaction task movement of the hand to close a signal key.

The onset of the fixation light thus served as a visual test stimulus delivered during a state of alert vigilance without directed visual attention. We have compared the responses of LS neurons to this target onset stimulus with those evoked by physically identical lights presented during the trial periods; i.e., in what we define as a state of directed visual attention. We collected for each neuron studied in this way a large number of trials so that we could select those in which the test stimulus at the trial onset appeared in the same retinotopic positions as those delivered during the trials.

A typical result is shown in Figure 8. The central panel shows the response of a parietal LS neuron to probe stimuli presented during the trial period at the four retinotopic locations indicated by the four solid circles on the axes of the visual fields shown to the upper left. There is an intense and sustained response with a sharp initial transient. The panel to the right shows the responses of this same cell to the initial appearance of the 

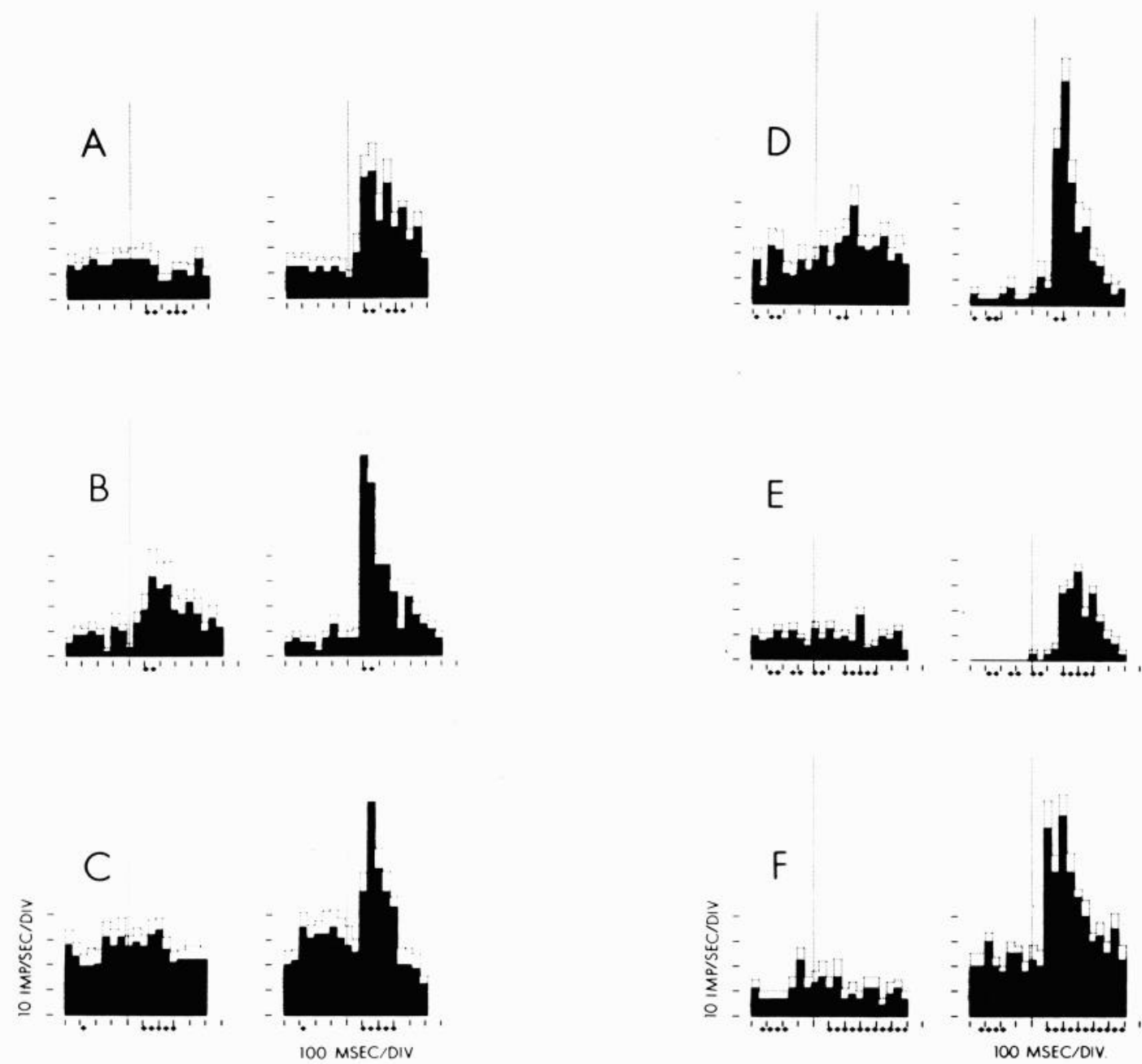

Figure 6. Comparisons of responses of parietal light-sensitive neurons to visual stimuli delivered in the task and no task states. Histograms of responses to physically identical visual stimuli delivered to similar retinotopic locations in the two states are shown; the left and right histograms of each pair sum responses in the no task and task states, respectively. For the method of analysis, see the legend to Figure 4 and the text. The differences in the populations of responses are significant at the $5 \%$ level for each of the 6 neurons. Facilitation ratios: $A, 14.8 ; B, 3.5 ; C, 9.1 ; D, 3.1$; $E, 11.8 ; F, 12.7$. All stimuli were $6^{\circ}$ squares of white light back-projected upon a $100^{\circ} \times 100^{\circ}$ area of a tangent screen placed $34 \mathrm{~cm}$ from the animal's eyes.

target light at the trial onset at the retinotopic positions indicated by the crosses on the axes of the visual field shown to the lower left. For these trials, eye position has been normalized to the center of these axes. The trials shown in the panel to the right in Figure 8 are arranged from above downward in order of decreasing latency of eye movement after the target onset. The onsets of eye movements are shown by the dashed line. The latencies vary from 400 to $150 \mathrm{msec}$, comparable to the range and variances of saccades evoked during the trial periods. The short latency neural response so clear in the central panel is greatly reduced in the records to the right. The eye movements evoked by this stimulus occurred long after the expected onset of the evoked response.

The stimuli used for the two states compared in Figure 8 were physically identical and delivered to similar retinotopic zones, but they differed in behavioral significance. For the trial tests of the middle panel, the monkey had to ignore the test stimuli, while at the end of the intertrial interval (right panel), he had to capture the target with foveating eye movements. Figure 9 shows the results obtained when the behavioral significance of the stimuli was identical in the two cases; i.e., when the test stimulus was also a saccade target in both the intertrial and the trial periods. The central panel shows the responses of the same cell as that of Figure 8 to the onset of the stimuli delivered to the same retinotopic positions as before, but now a foveating eye movement was made from the point of fixation to each test stimulus. These movements were triggered by the offset of the initial stimulus target that occurred synchronously with the onset of the test stimulus. Intense responses were evoked in the trial period by the test stimuli, responses that began well before the eye moved. The sustained component of the response, shown in Figure 8, was truncated here shortly after eye movement began. The right panel 


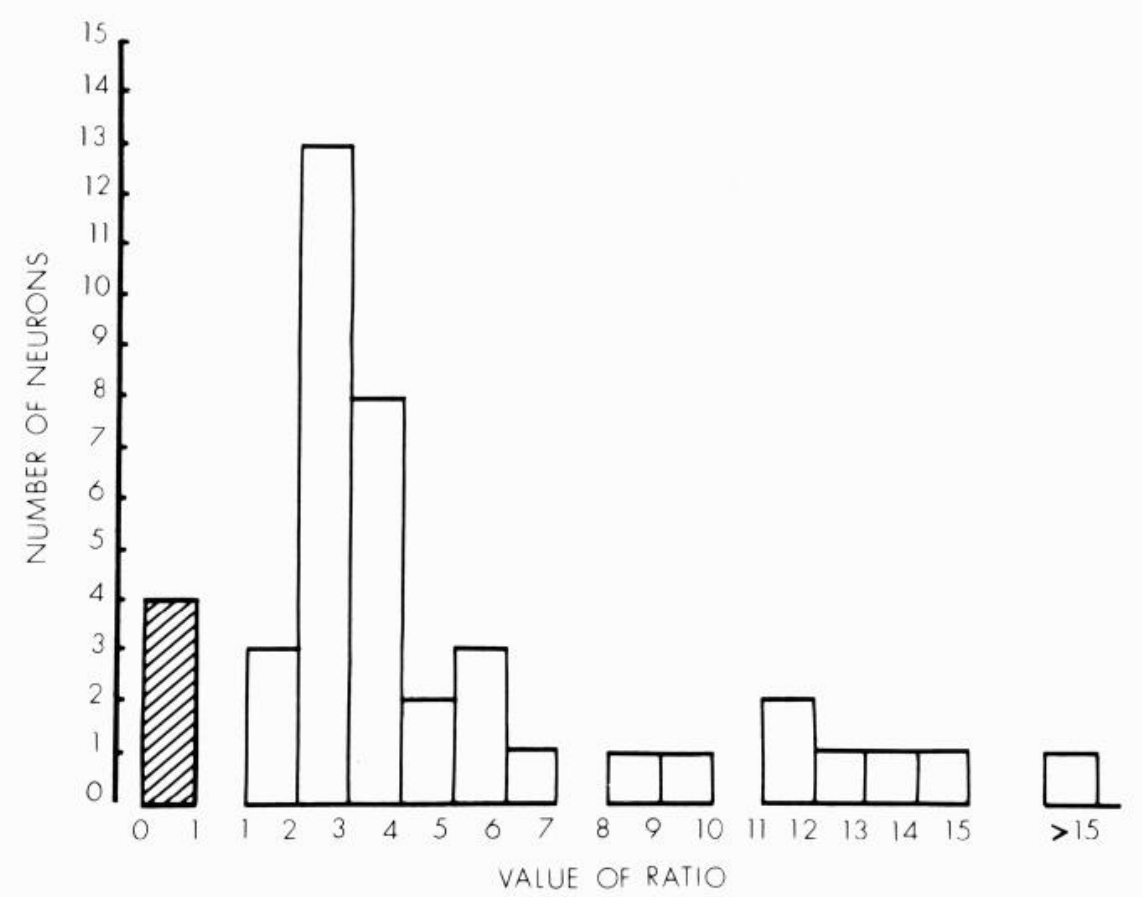

Figure 7. Distribution of facilitation ratios for parietal light-sensitive neurons. The facilitation ratio is that between the net increment in response evoked by a light stimulus in the state of interested fixation over that evoked by a physically identical and retinotopically similar stimulus delivered in the no task or the intertrial states. A total of 51 neurons showed ratios of 1.0 or greater, and of these, the difference was significant at the $5 \%$ level ( $t$ test) for 38 ; their values are plotted in the histogram. The ratio was fractional for 4 neurons indicated to the left: for them, the response was significantly greater in the no task state than during interested fixation.

of Figure 9 repeats that of Figure 8. It shows the striking difference in the responses evoked by the physically and spatially identical saccade targets in the two behavioral states.

The line of gaze was stationary for each case illustrated in Figure 9, and in each, a short latency eye movement was initiated to foveate the stimulus. The two states differ in that, during the trial period, the animal fixated a target light when the test stimulus appeared, whereas in the intertrial period, his gaze during eye pauses was directed upon the gray tangent screen with no target light. We show below, however, that the increase in excitability of LS neurons during attentive fixation occurs whether a target light is present or not. The only other difference in the two states compared in Figure 9 is the presence of attentive fixation in one and its absence in the other. We conclude that the act of attentive fixation is associated with an increase in the excitability of parietal LS neurons and that the latter is not due to a general arousal effect.

We have made comparisons of the type illustrated in Figures 8 and 9 for 92 parietal LS neurons. Sixty-eight resembled that illustrated in that responses to the initial appearance of the fixation target light were reduced or absent as compared to responses to identical stimuli delivered during the trial periods. The responses of the remaining 24 cells were either similar under all conditions (16 cells) or were slightly increased in the intertrial interval ( 8 cells). We have not been able to establish any correlation between the susceptibility of any LS neurons to this facilitatory effect and other response characteristics (i.e., with the temporal pattern of response, the sensitivity to movement, directionality, level of spontaneous activity, etc.). We have observed cells with response facilitation during attentive fixation in layers II through VI of the cortex; the 24 cells without facilitation were distributed preferentially in the supragranular layers.

Relation between the facilitation of attentive fixation and saccadic enhancement. It has been observed in two studies that the response of some parietal visual neurons may be increased when a test stimulus becomes a target for a saccade (Yin and Mountcastle, 1977; Robinson et al., 1978). An example is given in Figure 10. The responses in the left column were evoked by test stimuli during steady fixation and those of the central column were evoked by the same stimuli presented as saccade targets; both were delivered in the state of attentive fixation. The responses to the saccade stimuli are slightly more intense than are those to the test stimuli. The responses in the right column, by contrast, were evoked by physically identical saccade targets presented at the end of the intertrial periods in the same retinotopic areas as were the stimuli evoking the responses of the two adjacent panels. The difference in response to comparable saccadic targets seen between the central and the right panels illustrates the facilitative effect of attentive fixation. No correlation was observed between the presence or absence of the enhancement and the facilitation phe- 

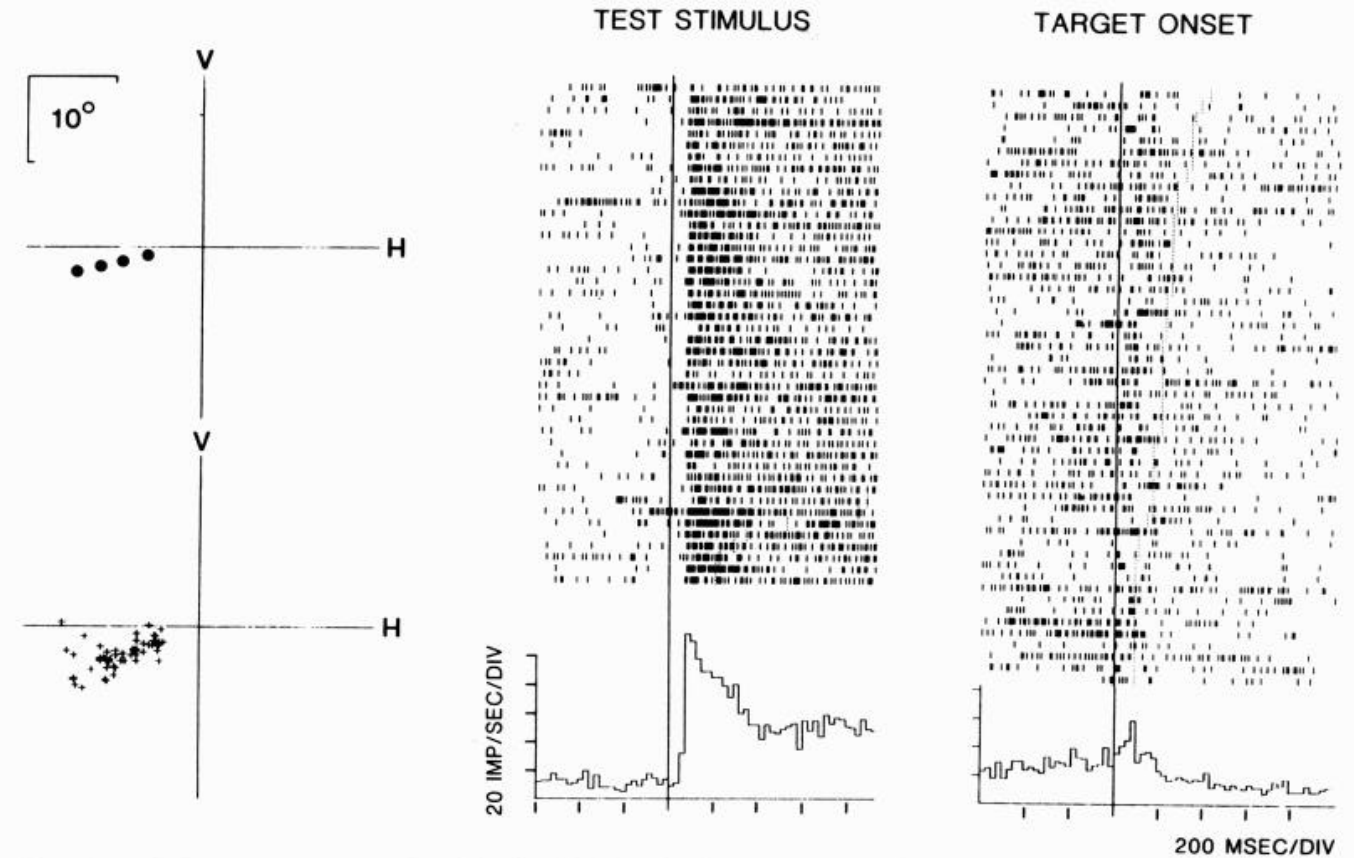

Figure 8. Facilitation of the response to visual stimulation during attentive fixation. Impulse replicas and the histogram of the central panel show the responses of a parietal light-sensitive neuron to the onset of the LED test stimulus during attentive fixation of a physically identical LED target. Those in the right panel show the responses of the same cell to the onset of the fixation target for trials when it appeared in retinotopic locations comparable to those of the test stimuli, during an eye pause in the intertrial interval, without attentive fixation. Rasters and histograms are aligned at the onset of the stimuli, indicated by the solid vertical lines. The dashed vertical line to the right of the solid vertical line in the right panel indicates the onset of eye movement after the target appearance. The axes to the left show the stimulus positions relative to the monkey's line of sight (center of axes) at the stimulus/target onset. Solid circles in the upper axes indicate the four positions tested while the monkey fixated a target, the test condition for the responses of the central panel. Crosses on the lower axes show positions of the fixation target relative to the monkey's line of sight at the instant of target appearance. The corresponding trials in the right panel were selected for coverage of a retinotopic area comparable to that of the four test stimuli, upper left axes. The response field of the cell included the entire contralateral (left) visual field out to at least $30^{\circ}$ from the fovea but excluded the central $3^{\circ}$. These are characteristics of parietal light-sensitive neurons with foveal sparing receptive fields.

nomena, but as seen in Figure 10, when both are present, the facilitation effects usually dominate.

The facilitation of parietal visual neurons during attentive fixation does not depend upon the presence of a target light. The responses of parietal visual neurons to visual stimuli have now been compared for three behavioral states, and the facilitation accompanying the state of attentive fixation has been described. However, task state A (see Fig. 1) differs from the no task state and the intertrial period, for in it, a second visual stimulus, the target light itself, is present in the visual field when the test stimulus is delivered but is not present in other states; i.e., our results might have been due to a form of sensory-sensory interaction. We designed a control experiment in which conditions would be more exactly comparable. Monkey 88 was trained to hold steady fixation of a spot on the tangent screen at which a fixation light had appeared briefly and disappeared and at which that light would later reappear and dim (see "Materials and Methods" and Fig. 1). Uncertainties in the length of the intertrial intervals and the foreperiods, combined with the EOG control described under "Materials and Methods," assured the continued attentive fixation of the proper locus on the tangent screen during the off period of the fixation light. Visual stimuli delivered during this period thus tested the excitability of parietal neurons under conditions that differed from those of the no task state only by the accompanying attentive fixation. We studied 15 parietal cells under these conditions; in every case, the response evoked in task state $A$ also was present in task state B. An example is given in Figure 11, which displays histograms of the responses of a parietal LS neuron evoked by stimuli delivered to a sensitive locus in the peripheral receptive field in the two task states and in the no task state. The test stimuli were physically identical in each case. The brisk responses evoked in task states A and B are similar, and each differs significantly from the small response evoked in the no task state. We have observed for some neurons that, even when similar responses are evoked from sensitive points in the receptive field, in task states A and B, the size or shape of the receptive field may be influenced to a small extent by the presence or absence of the fixation target light. We have not studied these more subtle aspects of the spatial interactions of two or more stimuli upon the properties of parietal visual neurons.

We examined 5 neurons in a reciprocal control experiment by placing a second light, identical to the fixation light of task state $\mathrm{A}$, on the tangent screen during the no task collection period. Thus, this second light and the 

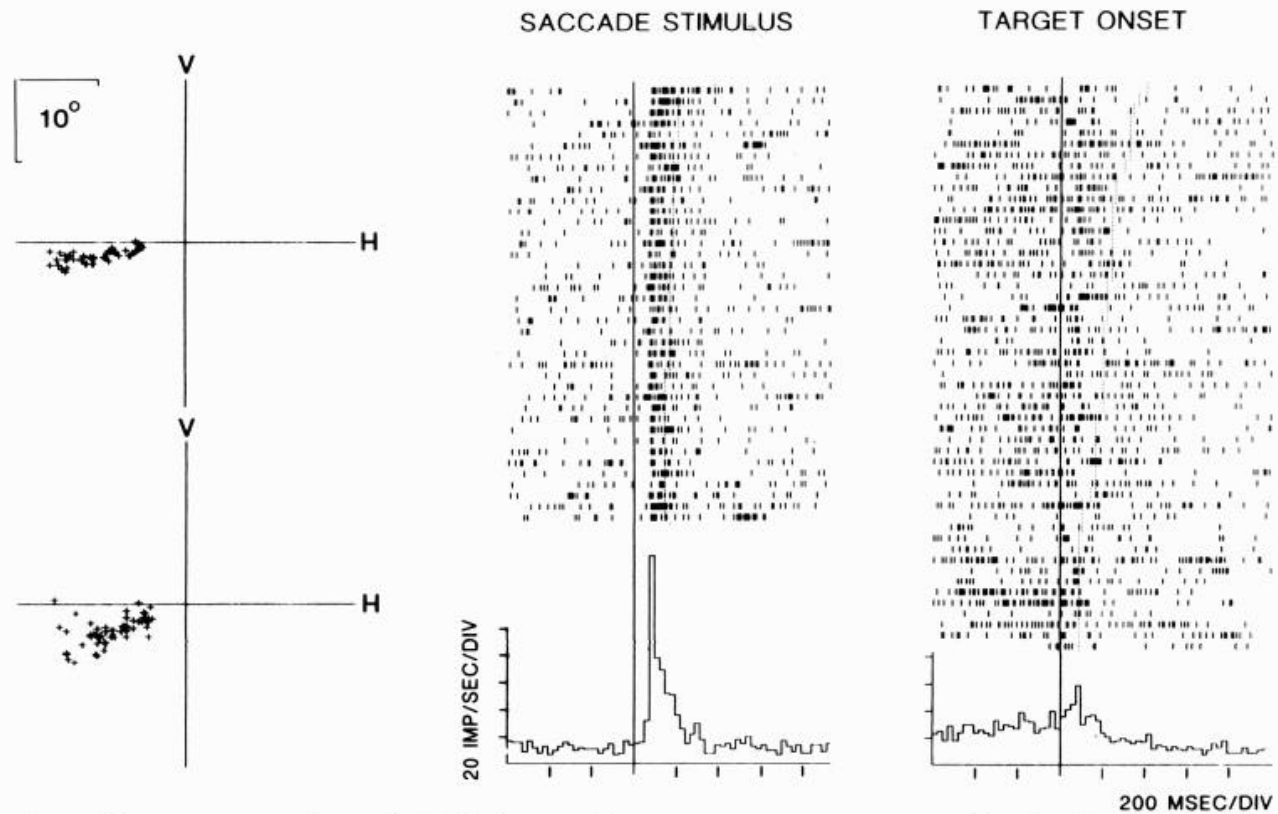

Figure 9. Facilitation of the response of a parietal light-sensitive neuron to a saccade stimulus during attentive fixation. Impulse replicas and the histogram of the central panel show responses of a cell to the onsets of visual stimuli to which saccadic eye movements were made from the then current positions of interested fixations of an identical LED target. The right panel and lower left axes repeat those of Figure 8, showing the responses of the cell to the onset of the fixation target to which saccadic eye movements were made. The onset of the saccade targets is marked by solid vertical lines; slanted dashed lines immediately to the right mark the onset of saccadic eye movement. Crosses on the upper left axes denote the initial positions of saccade stimuli relative to the monkey's line of sight as measured by the electro-oculogram. The actual positions were those shown in Figure 8 , upper left. The error in estimating retinotopic positions of targets (as in the lower left axes) is much smaller than that in the overall retinotopic field selected.

test light itself appeared in a random spatial relation to the line of gaze during eye pauses and to each other. The histograms of Figure 12 show that the presence of the second light within the visual field did not facilitate the response of the parietal LS neurons to test stimuli in the no task state.

We conclude that the facilitation observed in the state of directed or attentive fixation is independent of either the presence of a fixation light as target or the presence of a second light at some other position in the visual field. This facilitation is not due to sensory interaction but to the act of directed attention.

Darkness control. In our experiments, the animal faced a gray, homogeneous screen which subtended $127^{\circ}$ of horizontal and $150^{\circ}$ of vertical visual field. The contrast at the junctions of the screen and the walls of the recording chamber was small. It is unlikely that the differences in the retinotopic locations of background contours in the no task condition could account for the differential sensitivity that we observed between the task and no task states. Moreover, we studied 2 neurons in the task and the no task states in light and in total darkness. Both showed a significant and nearly identical increase in light sensitivity for the task state over the no task state in both the dark and lighted conditions. We conclude that the differences in light sensitivity do not result from visual interactions between the test stimuli and any static contrasts present in the experimental environment.

\section{Discussion}

Our observations indicate that the responses of parietal LS neurons to visual stimuli are conditional upon behavioral state. They are facilitated during what we define as task states as compared to the responses in the no task state and the intertrial interval. In the former, the animal worked at a visual detection task, directing his line of gaze toward a small target light whose dimming he had to detect for reward or toward a locus on the tangent screen at which the target light would later reappear, and then dim: the testing stimuli were passive in nature, for they never controlled behavior. The animal displayed signs of general alertness in the no task and the intertrial states, but he fixated no targets and performed no tasks. Care was taken to deliver physically identical testing stimuli to the same retinotopic locations in the states compared and to eliminate or equate the effects of eye movements upon the responses of parietal neurons.

Our hypothesis is that directed visual attention to foveated targets is accompanied by a facilitation of transmission in neural pathways linking the retinae and the parietal lobe and that this is a specific effect superimposed upon any increments in excitability associated with changes in the general state of arousal or alertness. Thus, stimuli in the visual surround gain a powerful representation in that cortical area thought to be an important part of the central mechanism for the re-direction of attention from one foveated object to a new one. Under 

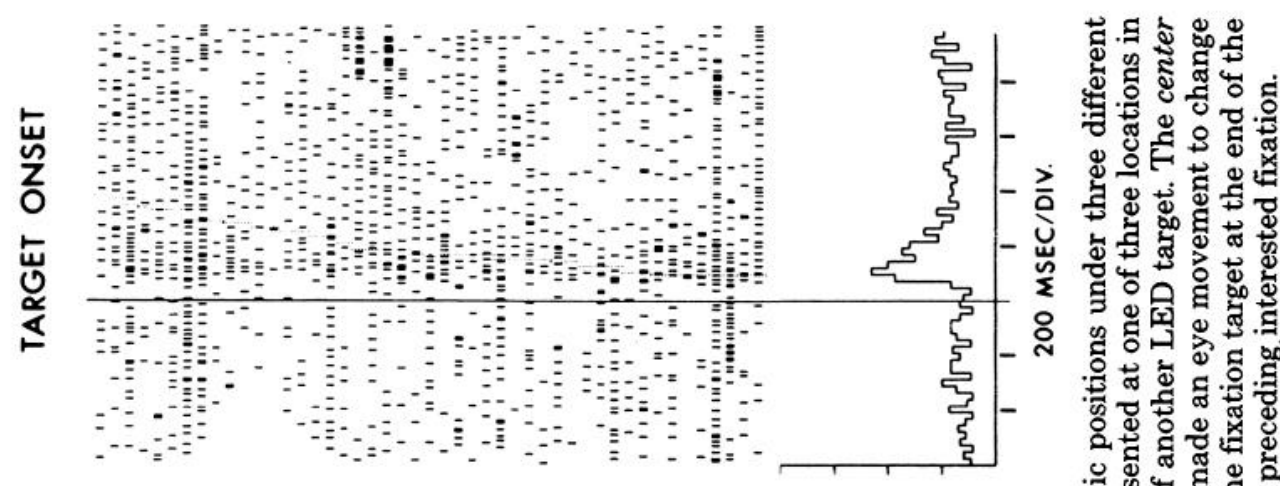

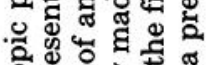

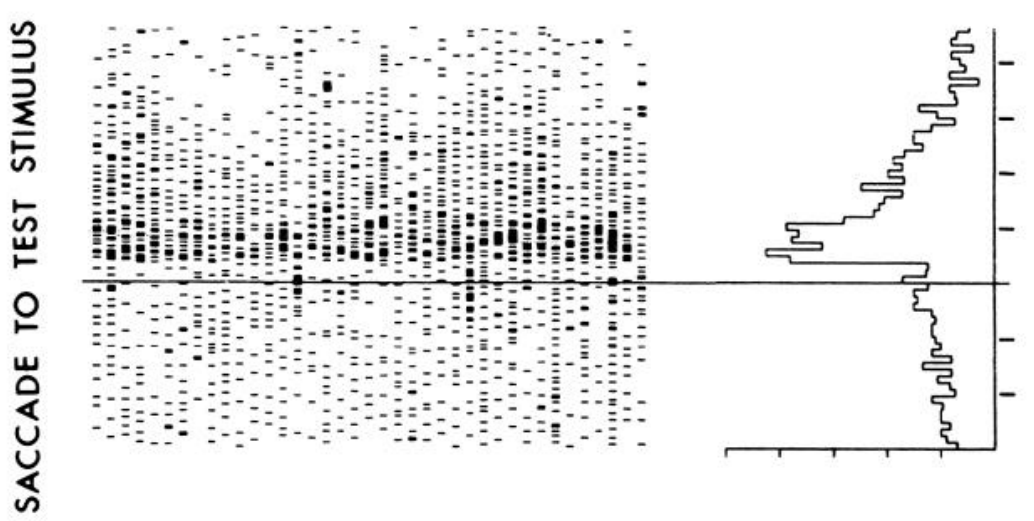

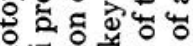

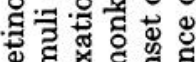

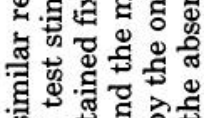

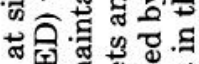

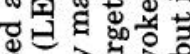

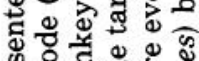

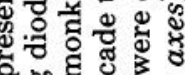

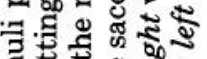

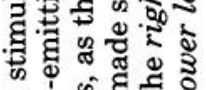

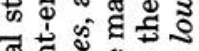

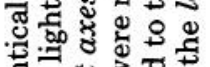

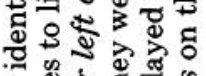
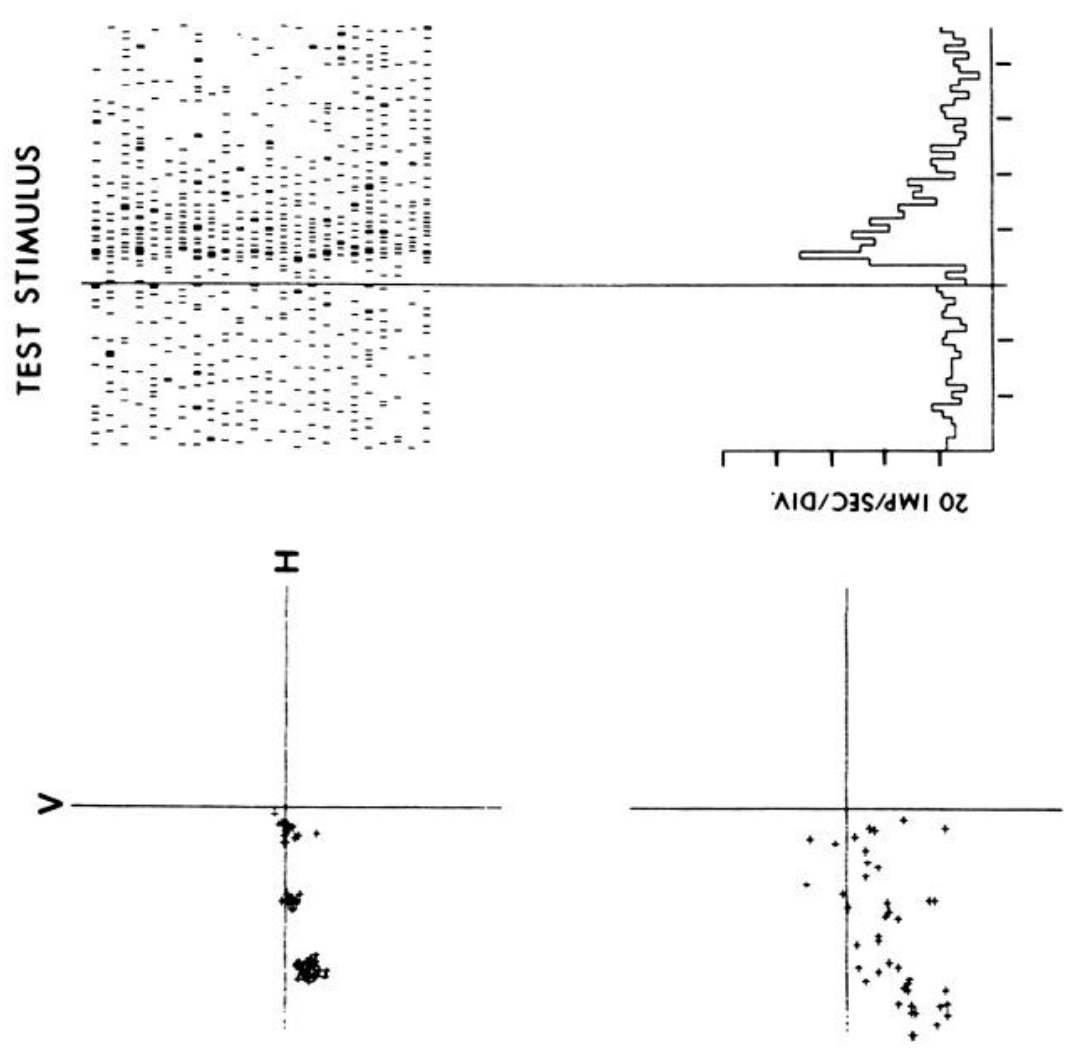

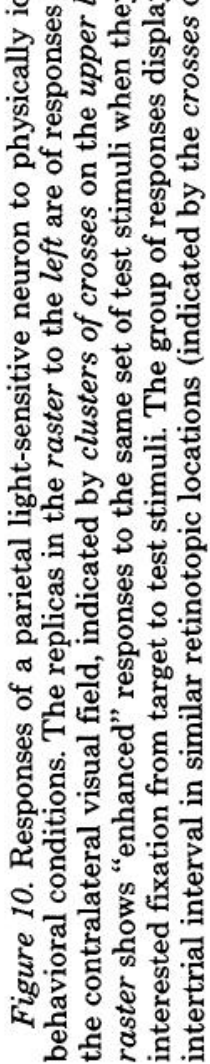


NO-TASK MODE

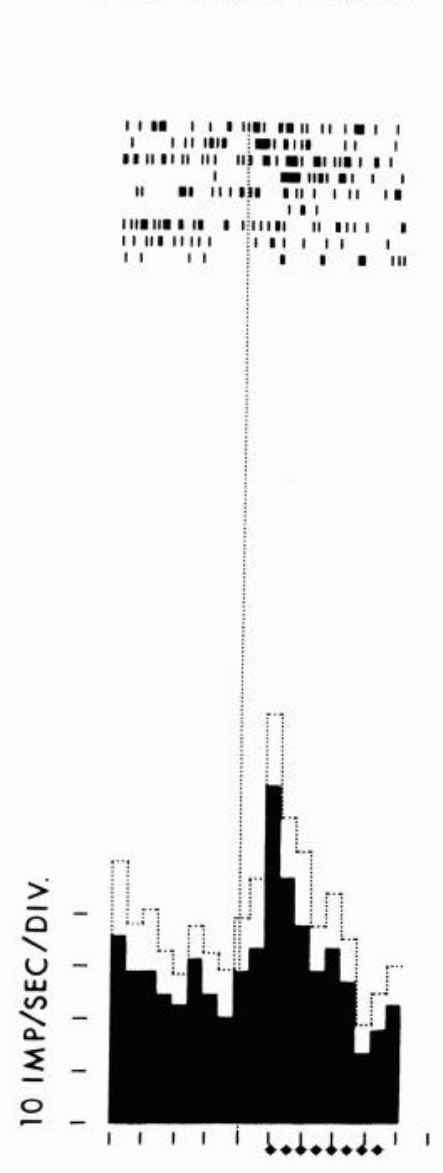

TASK MODE A

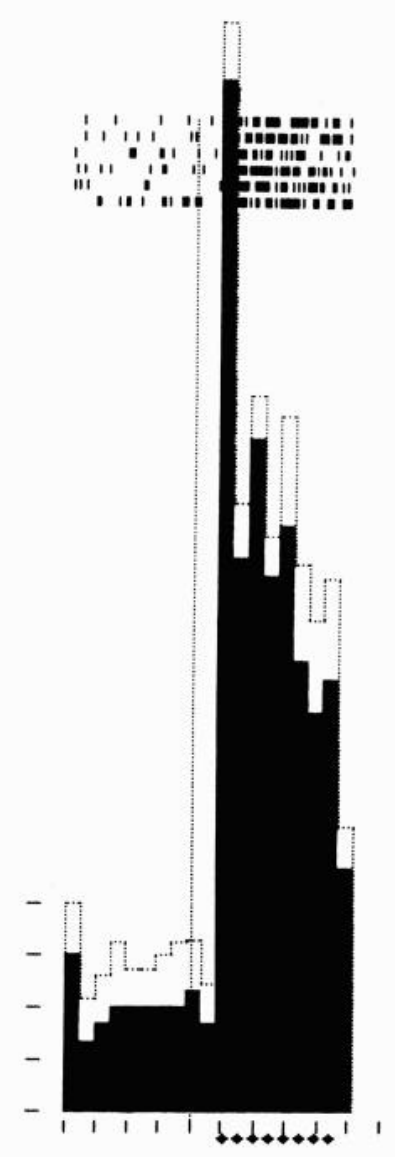

TASK MODE B

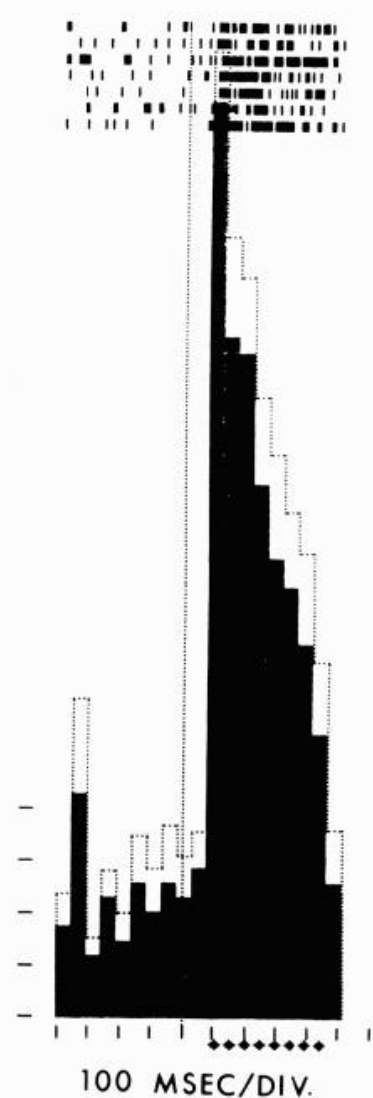

Figure 11. Comparison of responses of a parietal light-sensitive neuron to visual stimuli in the no task mode with those evoked during attentive fixation with (task mode $A$ ) and without (task mode $B$ ) a target light. The neuron subtended a receptive field extending from the central line of gaze upward $30^{\circ}$ along the vertical meridian, with the best responses given to a perifoveal $6^{\circ}$ $\times 6^{\circ}$ square flash. The central histogram sums responses evoked during task mode $A$ as the animal fixated a small target light. The histogram to the right shows that the responses were virtually identical when the animal fixated a blank locus on the tangent screen at which the target would later reappear in task mode $B$ (see Fig. 1). The left histogram sums the responses evoked in the no task mode. The facilitation ratio between the no task and the task mode $A$ populations of responses is 8.3 ; data analysis and display are described in the legend to Figure 4. Responses in task modes $A$ and $B$ also were studied in total darkness and were similar to those shown above. Dashed lines, 1 SEM for each bin of the histograms; diamonds, bins of the left histogram that differed significantly from similarly marked bins of the histograms at the center and right.

our experimental conditions, the line of fixation and that of attention are thought to be co-linear. Under other conditions, attention may be moved when the eyes are fixed, made to move toward an eccentric target before the eye moves to that target, or made to move in a direction opposite to that of an evoked eye movement (Posner, 1980).

The nature of the behavioral states compared. The behavioral states that we compared differ by the presence of attentive fixation in the trial states and its absence in the comparison states. It might be supposed, alternatively, that the states differ mainly in the level of arousal or attentiveness and that the incremented responses of parietal visual neurons during the trial state, even to unattended stimuli, may be attributed to the general increase in cortical excitability that accompanies states of heightened arousal. There are reasons to believe, however, that the general state of arousal is similar in the trial and intertrial periods. Monkeys in the latter, e.g.: (a) show signs of attentiveness comparable to those of the trial state; (b) make brisk spontaneous saccadic eye movements; (c) make visually evoked saccades to targets that signal the end of the intertrial interval at latencies comparable to those of saccades evoked by visual stimuli delivered in the trial period; and $(d)$ terminate the intertrial period, whose duration they cannot anticipate, with brisk reaction task movements. We conclude, therefore, that the rapid state transition which occurs at the end of the intertrial interval is characterized by the appearance of attentive fixation occurring against a background of a more or less steady state of arousal. This change is accomplished in no more than 600 to $700 \mathrm{msec}$, and perhaps much less, and is associated with a strong facilitation of parietal visual neurons. This facilitation does not depend upon the presence of a target light but upon the attentive fixation itself (Fig. 11).

If the level of arousal were the important factor in the facilitation observed, it would be expected to produce 
NO-TASK MODE
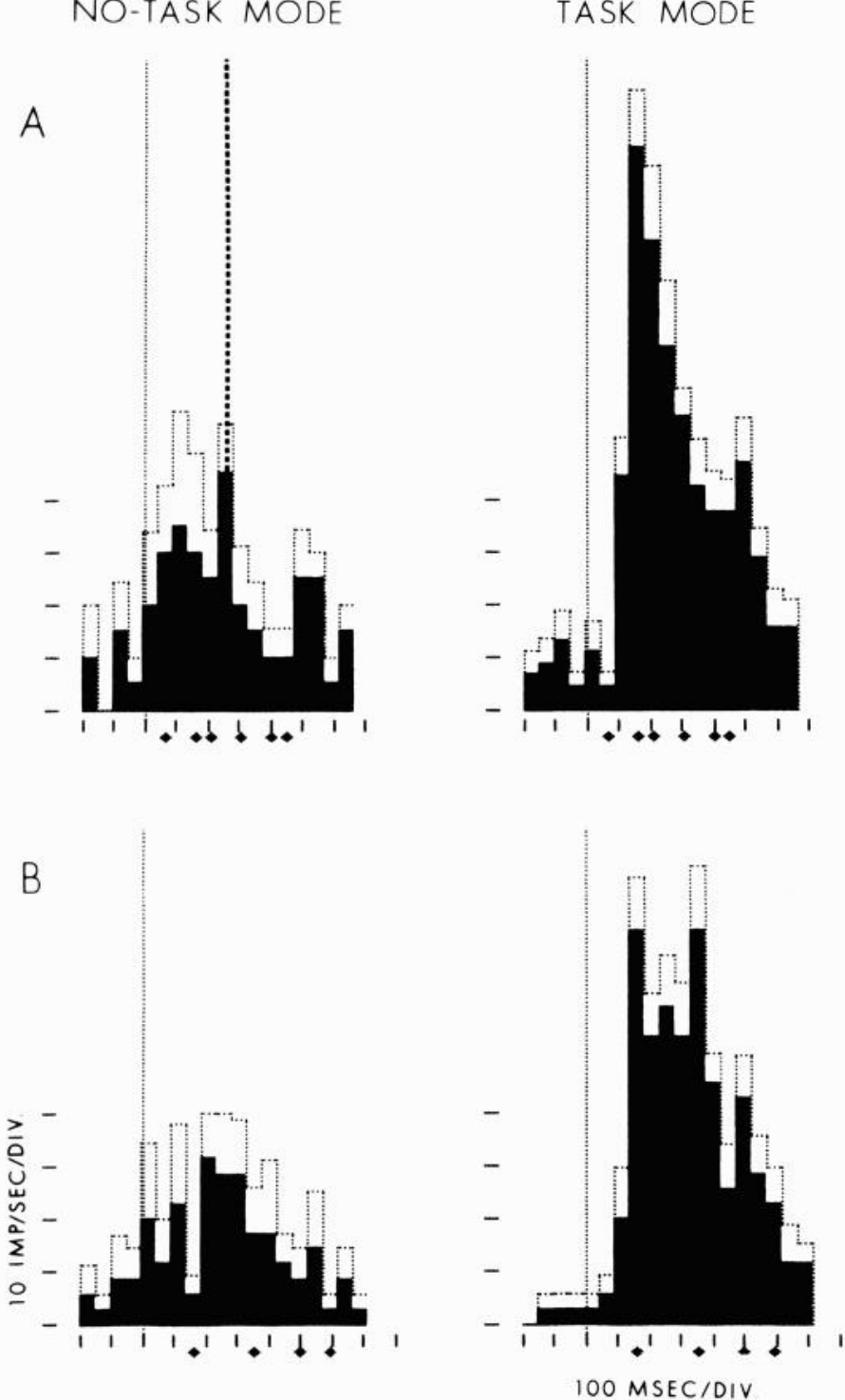

Figure 12. Evidence that the facilitation of parietal lightsensitive neurons does not depend upon the presence of the target light in the field. $A$, This neuron was related to a receptive field extending from $50^{\circ}$ above to $50^{\circ}$ below the line of fixation along the vertical meridian, sparing the immediate perifoveal region. The right histogram sums responses during task mode $A$ evoked during attentive fixation of a small laser spot target light by $6^{\circ} \times 6^{\circ}$ test flash centered $10^{\circ}$ above the line of gaze along the vertical meridian. The left histogram sums responses evoked by physically and retinotopically identical test flashes during the no task mode, with a second light identical to the laser spot target light now present in the field with each testing flash. Dashed vertical line left, the onset of the laser spot; heavy dashed line, the onset of the test flash; dashed vertical line right, the onset of the testing flash. $B$, This neuron related to the receptive field extending at least $50^{\circ}$ away from the point of fixation along each limb of both the vertical and the horizontal meridians but sparing the immediate perifoveal region. The right histogram sums responses to laser spot test stimuli placed $20^{\circ}$ above the target laser spot along the vertical meridian. The left histogram sums responses to physically and retinotopically identical test stimuli, with a second laser spot present elsewhere in the field. Dashed vertical lines, the onset of test stimuli. The selection and analysis of the data are described in the legend to Figure 4. Dashed lines above bars, 1 SEM for each cell of the histograms; diamonds, cells of the histograms that differ significantly in the pairs compared. differences between responses in the no task mode and those of the intertrial interval. We observed, however, that the differences in the responses of parietal visual neurons are just as great between the no task state and the trial period as between the intertrial period and the latter. We take the abrupt increase in light sensitivity that occurs with the transition from the intertrial to the trial period as evidence that the act of attentive fixation influences the excitability of parietal visual neurons in a specific way, independent of any accompanying changes in the level of arousal that may occur.

We have found no description of an experiment on the geniculostriate system in which the excitability of visual neurons was compared in waking monkeys with and without attentive fixation. The experiment most nearly comparable is that of Richmond and Wurtz (1981), who compared the response properties of visual neurons of the inferotemporal cortex in the waking, attentively fixating monkey with those evoked in the $\mathrm{N}_{2} \mathrm{O}$-anesthetized, neuromuscularly blocked state. They confirmed the observations of Gross et al. (1972) that, in the latter state, inferotemporal visual neurons are related to large receptive fields and often respond only to complex stimuli. Richmond and Wurtz (1981) observed that, in the attentively fixating monkey, inferotemporal visual neurons could be activated only by stimuli at or near the fixation point and then only weakly. The results obtained differ from our own, for attentive fixation appears to suppress the excitability of temporal LS neurons while facilitating that of parietal ones. The inference is that the influence of attentive fixation is specific and different for each of these cortical areas. Richmond and Wurtz also observed that the target light itself was important for the suppressive effect on inferotemporal neurons. This contrasts with our observation that the target light itself is not required for the facilitatory action of attentive fixation on the responses of parietal LS neurons.

The effects of attention upon central neural activity evoked by sensory stimuli, studied in waking animals. Studies in cats and monkeys of the effects of attention upon central neural activity evoked by sensory stimuli have shown that: (a) there is an increase in evoked responses when the animal transits from a non-performing to a task-performing state (Miller et al., 1972; Pfingst et al., 1977; Hyvarinen et al., 1980); (b) attention to a particular sensory modality facilitates neural responses in some cortical areas related to that modality (Ricci et al., 1967); (c) selective attention to a particular sensory attribute within a single modality selectively facilitates responses to stimuli with that attribute (Beaton and Miller, 1975; Benson and Heinz, 1978; Hocherman et al., 1976). These studies were made with protocols that differ from that which we used for comparison between the task and no task conditions: in the former, the test stimuli controlled behavior; in the latter, they were behaviorally irrelevant.

These findings indicate that the general facilitatory effect associated with transition from an idling to an attentive, task-performing state is small compared to the degree of facilitation of parietal LS neurons associated with attentive fixation. Moreover, it is clear that this general facilitation would occur, in our animal subjects, between the no task and the task-performing conditions and would be more or less uniform between the trial and 
the intertrial intervals within the latter. Yet we found the facilitatory effect of attentive fixation upon parietal LS neurons to be just as great for the trial/intertrial as for the trial/no task comparison. This lends further support to the conclusion that the mechanism of the facilitation associated with attentive fixation is specific for that behavioral state and, as far as is presently known, for parietal visual mechanisms.

The effect of selective attention upon evoked and event-related potentials in man. It is clear that attention influences sensory-evoked and event-related potentials recorded from the scalp in man. These electrical changes are considered to be signs of neural events related to sensory processing and perception (for reviews, see Naatanen, 1975; Naatanen and Michie, 1979a, b; Hillyard and Picton, 1979; Hillyard et al., 1978; Picton et al., 1978). Investigators of this subject emphasize the selective aspects of attention and have sought correlations between changes in electrical events and differential responses to attended versus unattended stimuli in paradigms in which, as far as possible, all other variables are controlled or eliminated. The results obtained appear generally the same for visual, auditory, and somatic sensory systems. The early electrical potentials evoked by sensory stimuli are unaffected by selective attention. The first change observed is an increase in the amplitude of a negative wave peaking at a latency of about $100 \mathrm{msec}$, called the $\mathrm{N}-1$, thought by some to be a sign of the central neural events involved in "stimulus set" selection (Broadbent, 1970). The change associated with selective attention is produced by a summation of an unchanged N-1 with a steady negative shift in transcortical potential resembling a contingent negative variation (Naatanen et al., 1978; Hansen and Hillyard, 1980). The mechanisms of this negative variation are unknown, but it appears from the selective attention studies that it can occur differentially both in regard to the sensory system and different "channels" within a system.

Our experiments were made in a different paradigm, which we label directed (or focused) attention. No differential response was required of the animal, and the paradigm was selective only in the general sense that he was required to withhold responses to all other stimuli and to maintain fixation of a single target. Our results differ from those expected on the basis of the human studies referred to above: attentive fixation of a target facilitated the responses of parietal visual neurons to unattended stimuli that did not control behavior. Our experimental circumstances are, however, exactly those known to be associated with widely distributed contingent negative variations of steady transcortical potentials (Naatanen and Michie, 1979b): a reaction task paradigm with a variable S1-S2 interval. We have no evidence whether such a negative variation occurred in our experiments.

On residual visual function in primates after lesions of the striate cortex: Relation to the parietal visual system. Humans with lesions of the striate cortex retain, or can recover with training, a residual capacity for some forms of visual behavior evoked by stimuli delivered in the blind, contralateral half-field, a capacity demonstrated best in the forced choice circumstance (Weiskrantz et al., 1974; Weiskrantz, 1980; Perenin, 1978; Per- enin and Jeannerod, 1978). All except one of these patients denied conscious visual experiences while operating correctly upon visual stimuli in their hemianopic field. This single patient, subject "G" of Barbur et al. (1980), the most intensively studied of all, detected stationary flashing stimuli in his "blind" field at intensities only $1.5 \mathrm{log}$ units above normal thresholds, showed increment thresholds 2 log units above normal, as well as spatial summation in accord with Ricco's law. He detected movement and the direction of real or apparent motion as do normal subjects. The subject failed to discriminate the orientations, shapes, or sizes of stimuli but projected his hand to touch stimuli in the blind field with mean errors only slightly above normal but with increased variability. Other subjects have shown a minimal capacity to make saccadic movements of the eyes to targets in the blind fields (Poppel et al., 1973). "G" reported no visual experience evoked by stimuli at his detection threshold; stronger stimuli elicited an experience of dark shadow, and still stronger ones produced an experience of a bright light. All other subjects denied any visual experience, even when, in the forced choice experiment, they demonstrated detections, discriminations, and visually guided movements with an accuracy comparable to that of "G."

Monkeys display similar retained capacities for visual function and visually guided behavior after removals of the striate cortex (Humphrey, 1974; Weiskrantz et al., 1977; Schilder et al., 1971, 1972; Pasik and Pasik, 1971). This capacity remains after lesions of areas 18 and 19 are added to one of the striate cortex but is then lost, in such a monkey, when an additional removal is made of the superior colliculus and pretectal areas (Keating, 1980).

These findings suggest that a portion of the visual system other than its geniculostriate component may be sufficient as the afferent pathway for certain types of visually guided behavior and for performance in visual discriminations and detections in the forced choice experiment, all without conscious perception. These capacities appear to depend upon the retinocollicular component of the visual system, and the majority of investigators have attributed the residual function to the superior colliculus and associated subcortical systems. Indeed, hemispherectomized humans show some residual visual capacity in the contralateral hemifield, but that which remains is small compared to that of humans with striate lesions (Perenin, 1978). This suggests that telencephalic components of the collicular system may be essential for the full complement of residual capacities in de-striate primates.

The inferior parietal lobule (area 7) of the monkey is a projection target of this "second" visual system, and the functional properties of its visual neurons match the remaining capacities for visual function of humans and monkeys after striate lesions (Motter and Mountcastle, 1981). The sensitivity of these cells to stimulus movement and direction matches the capacity of de-striate primates to detect movement and direction and to discriminate between velocities. The presence in the inferior parietal lobule of these visual neurons together with other sets thought to be important in visually guided projections of the arm and hand (Mountcastle et al., 1975) fits with the retained capacity of de-striate humans to make correct 
projections to targets in their blind fields. Moreover, all of these residual capacities were demonstrated as subjects attentively fixated foveal targets. These are just the circumstances which our present results show produce the greatest facilitation of the parietal visual system. We suggest that the inferior parietal lobule plays a role in the residual visual function of de-striate primates. An experiment to test this hypothesis directly has not been done (i.e., to examine the residual visual capacity of monkeys after removals of areas 17,18 , and 19 and the inferior parietal lobule). In normal primates, the facilitation with attentive fixation will exaggerate the response of the parietal visual system to attention-provoking. stimuli and lead to visually guided behavior executed, it may be inferred, without direct inference with or access to conscious visual perception.

Attentive fixation, eccentric vision, and the function of the parietal visual system. The human visual capacity is lower in the eccentric than in the central portion of the visual field, and the former is attenuated even further during attentive fixation of foveal targets (Bouma, 1978; Sanders, 1970; Webster and Haslerud, 1964). We assume that similar conditions occur in the rhesus monkey. There results in the human during attentive fixation a "functional visual field" centered on the fovea, in which the observer can carry out certain visual functions during fixations; it is about $10^{\circ}$ in diameter and its edges are defined by elevations in the luminance threshold and decreases in the ability to detect and to discriminate. The degree of this eccentric incapacity and the size of the functional visual field are influenced by the amount of information in the foveal fixation target and by the difficulty of both the foveal and eccentric visual tasks, especially when the later involves two or more targets with contextual background (Andriessen and Bouma, 1976; Engel, 1974; Ikeda and Takeuchi, 1975; Liebowitz and Appelle, 1969; Abernathy and Liebowitz, 1971). The functional field can be made to bulge asymmetrically in the direction of a displacement of attention when attention and the line of gaze are dissociated (Grindley and Townsend, 1968).

These facts suggest that, during attentive fixation and foveal work, there is a reduction or change in the nature of the central neural activity evoked by stimuli in the eccentric visual fields in parallel with the reduced capacity for eccentric vision measured in psychophysical experiments in humans. This change seems likely to occur at some level of the chain of neural operations leading to conscious visual perception. However, we found no published account of a study in waking monkeys designed to test this hypothesis by recording in striate or pre-striate cortex under appropriate experimental circumstances.

Our study of the parietal visual neurons yielded a result quite different from that expected: attentive fixation is accompanied by a facilitation, not a depression, of the responses of parietal visual neurons to visual stimuli. Moreover, we observed this facilitation to affect indiscriminately parietal visual neurons with eccentric, foveal, or bilateral receptive fields. One implication of our finding is that, under the conditions of our experiments, some components of the retinoparietal system may not function in the main stream of the neural processes leading to conscious visual perception as usually defined. A sec- ond implication is that the facilitated retinoparietal system may, during the hazardous act of restricting attention to objects fixated foveally, play a role in attracting attention to new, imperious, or threatening stimuli and in visually guided movement. On these grounds, the parietal visual mechanism appears to function as a part of the ambient rather than the focal visual systems as these were defined by Trevarthen (1968).

Neural mechanisms of the facilitation accompanying attentive fixation. Our observations provide no evidence bearing directly on this question, but they do suggest a number of the properties that such a control system may have: $(a)$ it may operate with a time course as short as 600 to $700 \mathrm{msec} ;(b)$ it may persist steadily for periods as long as $5 \mathrm{sec}$; but (c) it can oscillate repetitively from minimum to maximum with periods as short as our shortest trial + intertrial periods, 3 to $4 \mathrm{sec} ;(d)$ it can exert its effect differentially in regard to cortical areas; and $(e)$ it can be superimposed upon and, we believe, differentiated from the general arousal of the forebrain. Changes in cortical neuronal excitability associated with shifts in the level of arousal and/or attention are thought to reflect the influences of controlling systems originating in the central core of the brainstem. Among these are the monoaminergic nuclei that may influence telencephalic structures directly via their long and widely distributed axons. Alternatively, or concurrently, an ascending reticular system regulates cortical excitability by controlling thalamocortical elements or cortical neurons directly.

The locus coeruleus is the main source of a noradrenergic system which innervates all areas of the cerebral cortex directly in a widely divergent manner. It plays a role in regulating the sleep-wakefulness cycle and perhaps in other general functions as well (for reviews, see Amaral and Sinnamon, 1977; Moore and Bloom, 1979; Clark, 1979). Coeruleus neurons respond to a variety of sensory stimuli, particularly those with attention-provoking qualities (Foote and Bloom, 1979), and their discharge rates increase during arousal with desynchronization and dramatically so in the transition from quiet to attentive wakefulness (Chu and Bloom, 1973). Stimulation of the locus coeruleus produces an initial hyperpolarization of cortical neurons, followed by a prolonged potentiation of the responses of cortical neurons to other inputs. This effect of norepinephrine is attributed to activation of the adenyl cyclase system of the postsynaptic neurons.

There are reasons to question whether the coeruleus system can mediate the retinoparietal facilitation of attentive fixation. Firstly, its prolonged potentiation does not match the speed of change and rapid alteration of the states observed. Secondly, it is only with additional hypotheses that a differential effect of this system upon different cortical areas can be accounted for. And, thirdly, normal sleep-waking cycles and changes in excitability with attentive states survive near total destruction of the locus coeruleus and depletion of forebrain norepinephrine (Jones et al., 1977; Crow et al., 1978; Robinson et al., 1977).

It is known from the experiments of Magoun and Moruzzi that the mesencephalic reticular formation (MRF) plays an important role in regulating the excitability of the forebrain (see Moruzzi, 1972) and that the discharge rate of MRF neurons increases with increasing 
arousal (Steriade et al., 1979). A major projection of the MRF is upon the dorsal thalamus; and MRF stimulation, like behavioral arousal, facilitates transfer of neural activity through dorsal thalamic nuclei to the cerebral cortex (Malcolm et al., 1970; Maffei et al., 1965; Coenen and Vendrik, 1972; Steriade and Hobson, 1976; Steriade, 1980). The MRF also projects to the thalamic reticular nucleus, upon which its action is inhibitory (Fukuda and Iwama, 1971; Schlag and Waszak, 1971). Neurons of the thalamic reticular nucleus receive excitatory input from the collaterals of the thalamocortical and corticothalamic axons and are themselves inhibitory to the thalamocortical neurons of thalamic nuclei (Sumitomo et al., 1976; Dubin and Cleland, 1977; Singer, 1980). This facilitation of dorsal thalamic transfer by disinhibition via the reticular nucleus is central to the "gating" hypothesis of MRF control of cortical excitability discussed by Yingling and Skinner (Yingling and Skinner, 1977; Skinner and Yingling, 1977).

The effect of MRF stinulation upon cortical cells is excitatory (Steriade, 1980; Singer, 1979, 1980) and is distributed widely to all cortical areas examined, including parietal areas 5 and 7 in the cat (Steriade et al., 1978). Moreover, a second and direct MRF projection to the neocortex is believed to contribute also to cortical facilitation and to be cholinergic (Steriade, 1980; Singer, 1979, 1980). A differential gating mechanism of this type is a likely candidate for the control of the facilitation of the retinoparietal system during attentive fixation. The major questions remain: how can the neural mechanisms associated with attentive fixation engage this system, and how can the latter exert a differential effect upon cortical areas.

\section{References}

Abernathy, C. N., III, and H. W. Liebowitz (1971) The effect of feedback on luminance thresholds for peripherally presented stimuli. Percept. Psychophys. 10: 172-174.

Amaral, D. G., and H. M. Sinnamon (1977) The locus coeruleus: Neurobiology of a central noradrenergic nucleus. Prog. Neurobiol. 9: 147-196.

Andersen, R. A., and V. B. Mountcastle (1980) 'The direction of gaze influences the response of many light sensitive neurons of the inferior parietal lobule (area 7) in waking monkeys. Soc. Neurosci. Abstr. 6: 673 .

Andriessen, J. J., and H. Bouma (1976) Eccentric vision: Adverse interactions between line segments. Vision Res. 16: $71-$ 78.

Barbur, J. L., K. H. Ruddock, and V. A. Waterfield (1980) Human visual responses in the absence of the geniculo-calcarine projection. Brain 103: 905-928.

Beaton, R., and J. M. Miller (1975) Single cell activity in the auditory cortex of the unanesthetized, behaving monkey: Correlation with stimulus controlled behavior. Brain Res. 100: 543-562.

Benson, D. A., and R. D. Heinz (1978) Single unit activity in the auditory cortex of monkeys selectively attending left vs. right ear stimuli. Brain 159: 307-320.

Bouma, H. (1978) Visual search and reading: Eye movements and functional visual field: A tutorial review. In Attention and Performance VII, J. Requin, ed., pp. 115-140, Lawrence Erlbaum Associates, Hillsdale, NJ.

Broadbent, D. E. (1970) Stimulus set and response set: Two kinds of selective attention. In Attention: Contemporary Theory and Analysis, D. I. Motofsky, ed., pp. 51-60, Appleton-Century-Crofts, New York.
Chu, N. S., and F. E. Bloom (1973) Norepinephrine containing neurons: Changes in spontaneous discharge patterns during sleeping and waking. Science 179: 908-910.

Clark, T. K. (1979) The locus coeruleus in behavioral regulation: Evidence for behavior specific versus general involvement. Behav. Neural Biol. 25: 271-300.

Coenen, A. M. L., and A. J. H. Vendrik (1972) Determination of the transfer ratio of cat's geniculate neurons through quasiintracellular recordings and the relation with the level of alertness. Exp. Brain Res. 14: 227-242.

Crow, T. J., J. F. W. Deakin, S. E. File, A. Longden, and S. Wendlandt (1978) The locus coeruleus noradrenergic system-evidence against a role in attention, habituation, anxiety and motor activity. Brain Res. 155: 249-261.

Dubin, M. W., and B. G. Cleland (1977) The organization of visual inputs to interneurons of the lateral geniculate nucleus of the cat. J. Neurophysiol. 40: 410-427.

Engel, F. L. (1974) Visual conspicuity and selective background interference in eccentric vision. Vision Res. 14: 159-471.

Foote, S. L., and F. E. Bloom (1979) Activity of norepinephrine containing locus coeruleus neurons in the unanesthetized squirrel monkey. In Catecholamines: Basic and Clinical Frontiers, F. Usdin, ed., pp. 625-627, Pergamon, Elmsford, NY.

Fukuda, Y., and K. Iwama (1971) Reticular inhibition of internuncial cells in the rat lateral geniculate body. Brain Res. 35: $107-118$

Grindley, C. G., and V. Townsend (1968) Voluntary attention in peripheral vision and its effects on acuity and differential thresholds. Q. J. Exp. Psychol. 20: 11-19.

Gross, C. G., C. E. Rocha-Miranda, and D. B. Bender (1972) Visual properties of neurons in the inferotemporal cortex of the macaque. J. Neurophysiol. 35: 96-111.

Hansen, J. C., and S. A. Hillyard (1980) Endogenous brain potentials associated with selective auditory attention. Electroencephalogr. Clin. Neurophysiol. 49: 277-290.

Hillyard, S. A., and T. W. Picton (1979) Event related potentials and selective information processing in man. Prog. Clin. Neurophysiol. 6: 1-52.

Hillyard, S. A., T. W. Picton, and D. Regan (1978) Sensation, perception and attention: Analysis using ERP's. In Eventrelated Brain Potentials in Man, E. Callaway, P. Tueting, and S. H. Koslow, eds., pp. 223-321, Academic Press, New York.

Hocherman, S., D. A. Benson, M. H. Goldstein, H. E. Hefner, and R. D. Heinz (1976) Evoked unit activity in auditory cortex of monkeys performing a selective attention task. Brain Res. 117: 51-68.

Humphrey, N. K. (1974) Vision in a monkey without striate cortex: A case study. Perception 3: 241-255.

Hyvarinen, J., A. Poranen, and Y. Jokinen (1980) Influence of attentive behavior on neuronal responses to vibration in primary somatosensory cortex of the monkey. J. Neurophysiol. 43: 870-882.

Ikeda, M., and T. Takeuchi (1975) Influence of foveal load on the functional visual field. Percept. Psychophys. 8: 225-260.

Jones, B. E., S. T. Harper, and A. F. Halaris (1977) Effects of locus coeruleus lesions upon cerebral monoamine content, sleep-wakefulness states, and the response to amphetamine in the cat. Brain Res. 124: 473-496.

Keating, E. G. (1980) Residual spatial vision in the monkey after removal of striate and preoccipital cortex. Brain Res. 187: 271-290.

Liebowitz, H. W., and S. Appelle (1969) The effect of a central task on luminance thresholds for peripherally presented stimuli. Hum. Factors 11: 387-392.

Lynch, J. C., V. B. Mountcastle, W. H. Talbot, and T. C. T. Yin (1977) Parietal lobe mechanisms for directed visual attention. J. Neurophysiol. 40: 362-389. 
Maffei, L., G. Moruzzi, and G. Rizzolatti (1965) Influence of sleep and wakefulness on the response of lateral geniculate units to sine wave photic stimulation. Arch. Ital. Biol. 103: 596-608.

Malcolm, L., I. S. C. Bruce, and W. Burke (1970) Excitability of the lateral geniculate nucleus in the alert, non-alert and sleeping cat. Exp. Brain Res. 10: 283-297.

Miller, J. M., D. Sutton, B. Pfingst, A. Ryan, R. Beaton, and G. Gourevitch (1972) Single cell activity in the auditory cortex of rhesus monkeys: Behavioral dependency. Science 177: 449-451.

Moore, R. Y., and F. E. Bloom (1979) Central catecholamine neuron systems: Anatomy and physiology of the norepinephrine and epinephrine systems. Annu. Rev. Neurosci. 2: 113168.

Moruzi, G. (1972) The sleep-waking cycle. Rev. Physiol. Biochem. Exp. Pharmacol. 64: 1-165.

Motter, B. C., and V. B. Mountcastle (1980) Active directed gaze controls the excitability of the light sensitive neurons of the inferior parietal lobule in the waking monkey. Soc. Neurosci. Abstr. 6: 673.

Motter, B. C., and V. B. Mountcastle (1981) The functional properties of the light-sensitive neurons of the posterior parietal cortex studied in waking monkeys: Foveal sparing and opponent vector organization. J. Neurosci. 1: 3-26.

Mountcastle, V. B., J. C. Lynch, A. Georgopoulos, H. Skata, and C. Acuna (1975) Posterior parietal association cortex of the monkey: Command functions for operations within extrapersonal space. J. Neurophysiol. 38: 871-908.

Naatanen, R. (1975) Selective attention and evoked potentials in humans: A critical review. Biol. Psychol. 2: 237-307.

Naatanen, R., and P. T. Michie (1979a) Early selective attention effects on the evoked potential: A critical review and reinterpretation. Biol. Psychol. 8: 81-136.

Naatanen, R., and P. T. Michie (1979b) Different variants of endogenous negative brain potentials in performance situations: A review and classification. In Human Evoked Potentials; Applications and Problems, D. Lehman and E. Callaway, eds., pp. 251-267, Plenum Press, New York.

Naatanen, R., A. W. K. Gaillard, and S. Mantysalo (1978) Early selective attention effect on evoked potentials reinterpreted. Acta Psychol. (Amst.) 42: 313-329.

Pasik, T., and P. Pasik (1971) The visual world of monkeys deprived of striate cortex: Effective stimulus parameters and the importance of the accessory optic system. Vision Res. Suppl. 3: 419-435.

Perenin, M. T. (1978) Visual function within the hemianopic field following early cerebral hemidecortication in man. II. Pattern discrimination. Neuropsychologia 16: 696-708.

Perenin, M. T., and M. Jeannerod (1978) Visual function within the hemianopic field following early hemidecortication in man. I. Spatial localization. Neuropsychologia 16: 1-13.

Pfingst, B. E., T. A. O'Connor, and J. M. Miller (1977) Response plasticity of neurons in auditory cortex of the rhesus monkey. Exp. Brain Res. 29: 393-404.

Picton, T. W., K. B. Campbell, J. Baribeau-Braun, and G. B. Prouix (1978) The neurophysiology of human attention: $A$ tutorial review. In Attention and Performance VII, J. Requin, ed., pp. 429-467, Erlbaum Associates, Hillsdale, NJ.

Poppel, E., R. Held, and D. Frost (1973) Kesidual visual function after brain wounds involving central visual pathways in man. Nature 243: 295-296.

Posner, M. I. (1980) Orienting of attention. Q. J. Exp. Psychol. 32: $3-25$.

Ricci, G. F., F. Valassi, and I. Zamparo (1967) Electrophysiological study of visual cortical mechanisms activated during avoidance conditioning trials in macaques. Arch. Ital. Biol. 105: 413-435.

Richmond, B. J., and R. H. Wurtz (1981) Constriction of visual receptive fields of inferior temporal cortex neurons during visual fixation. Invest. Ophthalmol. Vis. Sci. (ARVO Suppl.) 20: 148.

Robinson, 'T. E., C. H. Vanderwolf, and B. A. Pappas (1977) Are the dorsal adrenergic bundle projections from the locus coeruleus important for neocortical or hippocampal activation? Brain Res. 138: 75-98.

Robinson, D. L., M. E. Goldberg, and G. B. Stanton (1978) Parietal association cortex in the primate: Sensory mechanisms and behavioral modulations. J. Neurophysiol. 41: 910932.

Sanders, A. F. (1970) Some aspects of the selective process in the functional visual field. Ergonomics 13: 101-117.

Schilder, P., T. Pasik, and P. Pasik (1971) Extrageniculostriate vision in the monkey. III. Demonstration of brightness discrimination. Brain Res. 32: 383-398.

Schilder, P., P. Pasik, and T. Pasik (1972) Extrageniculostriate vision in the monkey. III. Circle vs triangle and 'red vs green' discrimination. Exp. Brain Res. 14: 436-448.

Schlag, J., and M. Waszak (1971) Electrophysiological properties of units of the thalamic reticular complex. Exp. Neurol. 32: 79-97.

Singer, W. (1979) Central-core control of visual cortex function. In The Neurosciences: Fourth Study Program, F. O. Schmitt and F. G. Worden, eds., pp. 1093-1110, MIT Press, Cambridge, MA.

Singer, W. (1980) State dependent changes in ascending pathways affecting visual cortex functions. Neurosci. Res. Program Bull. 18: 91-100.

Skinner, J. E., and C. D. Yingling (1977) Central gating mechanisms that regulate event-related potentials and behavior. A neural model for attention. Prog. Clin. Neurophysiol. 1: 30-69.

Steriade, M. (1980) State-dependent changes in the activity of rostral reticular and thalamocortical elements. Neurosci. Res. Program Bull. 18: 82-91.

Steriade, M., and J. A. Hobson (1976) Neuronal activity during the sleep waking cycle. Prog. Neurobiol. 6: 155-376.

Steriade, M., A. Kitsikis, and G. Oakson (1978) Thalamic inputs and subcortical targets of cortical neurons in areas 5 and 7 of cat. Exp. Neurol. 60: 420-442.

Sleriade, M., A. Kitsikis, N. Robert, and G. Oakson (1979) The midbrain reticular core and related diencephalic areas: Neuronal organization and discharge properties during shifts in states of vigilance. In The Reticular Formation Revisited, J. A. Hobson and M. A. B. Brazier, eds., Raven Press, New York.

Sumitomo, I. Y., M. Nakamura, and K. Iwana (1976) Location and function of the so-called interneurons of the rat lateral geniculate body. Exp. Neurol. 51: 110-123.

Trevarthen, C. B. (1968) Two mechanisms of vision in primates. Psychol. Forsch. 31: 299-337.

Webster, R. G., and G. M. Haslerud (1964) Influence on extreme peripheral vision of attention to a visual or auditory task. $\mathrm{J}$. Exp. Psychol. (Hum. Percept.) 68: 269-272.

Weiskrantz, L. (1980) Varieties of residual experience. Q. J. Exp. Psychol. 32: 365-386.

Weiskrantz, L., E. K. Warrington, M. D. Sanders, and J. Marshall (1974) Visual capacity in the hemianopic field following a restricted occipital ablation. Brain 97: 709-728.

Weiskrantz, L., A. Cowey, and C. Passingham (1977) Spatial responses to brief stimuli by monkeys with striate cortex ablations. Brain 100: 655-670.

Yin, T. C. T., and V. B. Mountcastle (1977) Visual input to the visuomotor mechanisms of the monkey's parietal lobe. Science 197: 1381-1383.

Yingling, C. D., and J. E. Skinner (1977) Gating of thalamic input to cerebral cortex by nucleus reticularis thalami. Prog. Clin. Neurophysiol. 1: 70-96. 